\title{
On Determinant Expansions for Hankel Operators
}

https://doi.org/10.1515/conop-2020-0002

Received February 11, 2019; accepted January 20, 2020

\begin{abstract}
Let $w$ be a semiclassical weight that is generic in Magnus's sense, and $\left(p_{n}\right)_{n=0}^{\infty}$ the corresponding sequence of orthogonal polynomials. We express the Christoffel-Darboux kernel as a sum of products of Hankel integral operators. For $\psi \in L^{\infty}(i \mathbb{R})$, let $W(\psi)$ be the Wiener-Hopf operator with symbol $\psi$. We give sufficient conditions on $\psi$ such that $1 / \operatorname{det} W(\psi) W\left(\psi^{-1}\right)=\operatorname{det}\left(I-\Gamma_{\phi_{1}} \Gamma_{\phi_{2}}\right)$ where $\Gamma_{\phi_{1}}$ and $\Gamma_{\phi_{2}}$ are Hankel operators that are Hilbert-Schmidt. For certain $\psi$, Barnes's integral leads to an expansion of this determinant in terms of the generalised hypergeometric $2 m F_{2 m-1}$. These results extend those of Basor and Chen [2], who obtained ${ }_{4} F_{3}$ likewise. We include examples where the Wiener-Hopf factors are found explicitly.
\end{abstract}

Keywords: orthogonal polynomials, special functions, Wiener-Hopf, linear systems

MSC: 47B35

\section{Introduction}

Definition 1.1. (i) Let $\phi \in L^{2}(0, \infty)$. Then the Hankel operator with scattering function $\phi$ is the integral operator

$$
\Gamma_{\phi} f(x)=\int_{0}^{\infty} \phi(x+y) f(y) d y,
$$

which is defined on a dense linear subspace of $L^{2}(0, \infty)$. (The term scattering function is not to be confused with symbol function.)

(ii) Let $v \in \ell^{2}(\mathbb{N} \cup\{0\})$. Then the Hankel matrix corresponding to $v$ is $[v(j+k)]_{j, k=0}^{\infty}$, which gives a densely defined operator in $\ell^{2}(\mathbb{N} \cup\{0\})$. (The connection between Hankel matrices and integral operators is discussed in $[29$, p. 53].)

Given a trace class Hankel operator $\Gamma$, the spectrum consists of 0 and a sequence of eigenvalues $\lambda_{j}$, listed according to algebraic multiplicity, such that $\sum_{j=0}^{\infty}\left|\lambda_{j}\right|$ converges. Then we define the Fredholm determinant of $\Gamma$ by $\operatorname{det}(I+\Gamma)=\prod_{j=0}^{\infty}\left(1+\lambda_{j}\right)$. For Hilbert-Schmidt $\Gamma$, we define the Carleman determinant by $\operatorname{det}_{2}(I+\Gamma)=$ $\prod_{j=0}^{\infty}\left(\left(1+\lambda_{j}\right) e^{-\lambda_{j}}\right)$. The purpose of the present paper is to compute Fredholm determinants such as $\operatorname{det}\left(I+\Gamma_{\phi}\right)$, using operator theory and tools from linear systems.

We also obtain results relating to finite Hankel determinants, which arise as follows. Let $w_{0}(x)$ be a continuous, positive and integrable weight on $(0, b)$. Then we can take $Z_{b}>0$ such that

$$
Z_{b}^{-1} \prod_{1 \leq j<k \leq n}\left(x_{j}-x_{k}\right)^{2} \prod_{j=1}^{n} w_{0}\left(x_{j}\right) d x_{j}
$$

${ }^{\star}$ Corresponding Author: Gordon Blower: Department of Mathematics and Statistics, Lancaster University, Lancaster, LA14YF, United Kingdom, E-mail: g.blower@lancaster.ac.uk

Yang Chen: Department of Mathematics, University of Macau, Avenida da Universidade, Taipa, Macau, China 
gives a probability measure on $(0, b)^{n}$. In (1.4), we identify $Z_{b}$ with a Hankel determinant.

For a bounded and measurable function $f: \mathbb{R} \rightarrow \mathbb{C}$, we define the linear statistic $\sum_{j=1}^{n} f\left(x_{j}\right)$ and consider the exponential moment generating function

$$
\mathbb{E} e^{-\sum f}=\frac{\int_{(0, b)^{n}} \exp \left(-\sum_{j=1}^{n} f\left(x_{j}\right)\right) \prod_{1 \leq j<k \leq n}\left(x_{j}-x_{k}\right)^{2} \prod_{j=1}^{n} w_{0}\left(x_{j}\right) d x_{j}}{\int_{(0, b)^{n}} \prod_{1 \leq j<k \leq n}\left(x_{j}-x_{k}\right)^{2} \prod_{j=1}^{n} w_{0}\left(x_{j}\right) d x_{j}},
$$

which is the ratio of two Hankel determinants. In particular, with $f(x)=-\log (\lambda-x)$, we have $p_{n}(\lambda)=\mathbb{E} \prod_{j=1}^{n}(\lambda-$ $x_{j}$ ), which is a monic polynomial of degree $n$. Moreover, Heine [15] showed that $\left(p_{n}(\lambda)\right)_{n=0}^{\infty}$ is the sequence of monic orthogonal polynomials with respect to the weight $w_{0}$. We introduce $h_{j}=\int p_{j}(x)^{2} w(x) d x$. Then the Hankel determinant

$$
D_{n}\left[w_{0}\right]=\operatorname{det}\left[\int_{(0, b)} x^{j+k} w_{0}(x) d x\right]_{j, k=0}^{n-1}
$$

satisfies

$$
D_{n}\left[w_{0}\right]=\prod_{j=0}^{n-1} h_{j},
$$

and $Z_{b}=D_{n}\left[w_{0}\right]$. In section 3, we consider how Fredholm determinants are related to finite Hankel determinants $\operatorname{det}[v(j+k)]_{j, k=0}^{n-1}$ when the weight $w_{0}$ is semiclassical in Magnus's sense [24]. Our results continue the analysis by Tracy and Widom [40].

The function

$$
K(x)=\frac{\gamma \sin \pi x}{\pi \sinh \gamma x} \quad(x \in \mathbb{R}, \gamma>0)
$$

is even, integrable and of rapid decay at infinity and has Fourier transform

$$
F(\xi)=\frac{\sinh \left(\pi^{2} / \gamma\right)}{\cosh \left(\pi^{2} / \gamma\right)+\cosh (\pi \xi / \gamma)},
$$

as in [13, p. 31, 1.9(14)]. The Wiener-Hopf operator on $L^{2}(0, \infty)$ is $W(F): f(x) \mapsto \int_{0}^{\infty} K(x-y) f(y) d y$. The Wiener-Hopf factorization

$$
1-F(\xi)=\psi_{-}(i \xi) \psi_{+}(i \xi)
$$

was considered by Basor and Chen [2], who obtained various identities for determinants of related Hankel operators on $L^{2}(0, \infty)$. The following integral plays a central role in their analysis

$$
\int_{-i \infty}^{i \infty}\left(\frac{\Gamma(a+s) \Gamma(1-a+s) \Gamma(b-s) \Gamma(1-b-s)}{\Gamma(b+s) \Gamma(1-b+s) \Gamma(a-s) \Gamma(1-a-s)}-1\right) z^{s} \frac{d s}{2 \pi i},
$$

where $\Gamma$ is Euler's gamma function, and $a$ and $b$ are real. Integrals of this form, except without the summand -1 , were used by Mellin, Barnes and Meier [12, p. 49] to develop theories of special functions; see (7.8). In section four, we introduce an algebra $\mathcal{C}_{2}$ of complex functions on a strip containing $i \mathbb{R}$ such that each invertible $\psi \in \mathcal{C}_{2}$ has a Wiener -Hopf factorization $\psi(i \xi)=\psi_{-}(i \xi) \psi_{+}(i \xi)$, and we consider the Wiener-Hopf operator $W(\psi)$ of $L^{2}(0, \infty)$ with symbol $\psi$. Then in section 5 , we consider the functions

$$
\begin{array}{ll}
\phi_{1}(x)=\frac{1}{2 \pi} \int_{-\infty}^{\infty}\left(\frac{\psi_{-}(i \xi)}{\psi_{+}(i \xi)}-1\right) e^{-i \xi x} d \xi & (x>0) \\
\phi_{2}(x)=\frac{1}{2 \pi} \int_{-\infty}^{\infty}\left(\frac{\psi_{+}(-i \xi)}{\psi_{-}(-i \xi)}-1\right) e^{-i \xi x} d \xi & (x>0)
\end{array}
$$

and the Hankel integral operators $\Gamma_{\phi_{1}}$ and $\Gamma_{\phi_{2}}$. The main Theorem 5.1 gives sufficient conditions for validity of the formula

$$
1 / \operatorname{det} W(\psi) W\left(\psi^{-1}\right)=\operatorname{det}\left(I-\Gamma_{\phi_{1}} \Gamma_{\phi_{2}}\right),
$$


along with sufficient conditions for the Hankel operators to be self-adjoint.

Self-adjoint bounded Hankel operators have been characterized up to unitary equivalence by the results of [25]. The methods of [25] emphasized the importance of linear systems, and in the current paper, linear systems are used to obtain expansions of the Fredholm determinant $\operatorname{det}\left(I-\Gamma_{\phi_{1}} \Gamma_{\phi_{2}}\right)$. In section 6, we consider Wiener-Hopf factorizations which lead to Barnes's integrals as in (1.9), so that $\phi_{1}$ and $\phi_{2}$ have explicit expansions in terms of exponential bases. When interpreted with suitable linear systems, these formulas give expansions of $\operatorname{det}\left(I-\Gamma_{\phi_{1}} \Gamma_{\phi_{2}}\right)$ in terms of the generalised hypergeometric function ${ }_{2 m} F_{2 m-1}$. These results extend those of Basor and Chen [2], who obtained ${ }_{4} F_{3}$ likewise. In sections 7 and 8 , we make specific choices of $\psi$ and interpret our results in particular examples.

\section{Linear systems and associated Hankel operators}

The results of this section enable us to use linear system methods to compute Fredholm determinants of Hankel operators. For a complex separable Hilbert space $H$, we let $\mathcal{L}(H)=\mathcal{L}^{\infty}(H)$ be the space of bounded linear operators on $H$ with $\|T\|$ the usual operator norm of $T \in \mathcal{L}(H)$, and $\mathcal{L}^{1}(H)$ the ideal of trace class operators; then for $1 \leq p<\infty$, let $\mathcal{L}^{p}(H)$ be the ideal of operators such that the Schatten $p$-norm $\|T\|_{\mathcal{L}^{p}(H)}=$ $\left(\operatorname{trace}\left(T^{\dagger} T\right)^{p / 2}\right)^{1 / p}$ is finite, where $T^{\dagger}$ denotes the adjoint of $T$.

The Mellin transform $f^{\star}(s)=\int_{0}^{\infty} x^{s-1} f(x) d x$ gives a unitary transformation $f(x) \mapsto f^{\star}(i \xi+1 / 2) / \sqrt{2 \pi}$ from $L^{2}(0, \infty) \rightarrow L^{2}(i \mathbb{R})$. Let $\mathbb{C}_{+}=\{z \in \mathbb{C}: \operatorname{Re} z>0\}$ be the right half-plane and let $H^{2}\left(\mathbb{C}_{+}\right)$be the Hardy space of holomorphic functions $f$ on $\mathbb{C}_{+}$such that $\sup _{x>0} \int_{-\infty}^{\infty}|f(x+i \xi)|^{2} d \xi$ is finite. By the Paley-Wiener Theorem, the Mellin transform gives a unitary transformation $L^{2}(0, \infty) \rightarrow L^{2}(i \mathbb{R})$ that restricts to the orthogonal subspaces $L^{2}(0,1) \oplus L^{2}(1, \infty) \rightarrow H^{2}\left(\mathbb{C}_{+}\right) \oplus H^{2}\left(\mathbb{C}_{-}\right)$.

Let $L_{j}(x)=(j !)^{-1} e^{x}(d / d x)^{j}\left(x^{j} e^{-x}\right)$ be the Laguerre polynomial of order 0 and degree $j$; then $\left(e^{-x / 2} L_{j}(x)\right)_{j=0}^{\infty}$ gives an orthonormal basis of $L^{2}(0, \infty)$. Taking the Laplace transform of the sequence $\left(e^{-x / 2} L_{j}(x)\right)_{j=0}^{\infty}$, we obtain an orthonormal basis for the space $H^{2}\left(\mathbb{C}_{+}\right)$, namely

$$
\left(\frac{(\lambda-1 / 2)^{n}}{\sqrt{2 \pi}(\lambda+1 / 2)^{n+1}}\right)_{0}^{\infty}
$$

With $\mathbb{N}=\{1,2, \ldots\}$, we introduce the standard Hilbert sequence space $\ell^{2}(\mathbb{N} \cup\{0\})$, with the standard orthonormal basis $\left(e_{n}\right)$ and introduce the usual shift operator by the operation $S e_{n}=e_{n+1}$ on $\ell^{2}(\mathbb{N} \cup\{0\})$. There is a unitary map $H^{2}\left(\mathbb{C}_{+}\right) \rightarrow \ell^{2}(\mathbb{N} \cup\{0\})$. We have unitary maps between the Hilbert spaces

$$
\begin{array}{ccc}
L^{2}(0,1) & \rightarrow & H^{2}\left(\mathbb{C}_{+}\right) \\
\downarrow & \nearrow & \downarrow \\
L^{2}(0, \infty) & \rightarrow & \ell^{2}(\mathbb{N} \cup\{0\})
\end{array},
$$

where the top arrow is the Mellin transform, the maps down on the left is the change of variables $x=e^{-\xi}$ for $0<x<1$ and $\xi>0$, and the bottom arrow across is the expansion in terms of the Laguerre basis. The diagonal arrow is the Laplace transform, and the right downward arrow is given by expansion with respect to $(2.1)$.

There are several equivalent expressions for the Hilbert-Schmidt norm of Hankel operators that appear here. Suppose that $\phi_{1}, \phi_{2} \in L^{2}(0, \infty)$, and extend them to $L^{2}(-\infty, \infty)$ by letting $\phi_{j}(u)=0$ for all $u<0$. Then by a simple Fourier transform calculation as in [5].

$$
\int_{-2 \alpha}^{2 \alpha}|u| \phi_{1}(u) \phi_{2}(u) d u=\int_{-\infty}^{\infty} \int_{-\infty}^{\infty} 4\left(\frac{\sin 2(x-y)}{x-y}-\frac{\sin ^{2}(x-y)}{(x-y)^{2}}\right) \hat{\phi}_{1}\left(\frac{x}{\alpha}\right) \hat{\phi}_{2}\left(\frac{y}{\alpha}\right) \frac{d x d y}{(2 \pi)^{2}} .
$$

Let $C_{c}^{\infty}$ be the space of infinitely differentiable functions that have compact support. 
Lemma 2.1. (Basor, Tracy [6]) Suppose momentarily that $f \in C_{c}^{\infty}$ is real and even, so $f(x)=f(-x)$. Then the Mellin transform $f^{\star}$ and the Fourier cosine transform $C(f)(x)=\sqrt{(2 / \pi)} \int_{0}^{\infty} \cos (x y) f(y) d y$ satisfy

$$
\frac{1}{2 \pi} \int_{-\infty}^{\infty}\left|f^{\star}(i y)\right|^{2} y \tanh (\pi y) d y=\int_{0}^{\infty} x(C(f)(x))^{2} d x .
$$

Proof. The fractional derivative

$$
D^{1 / 2} f(x)=\frac{1}{\sqrt{\pi}} \frac{d}{d x} \int_{x}^{\infty} \frac{f(u)}{\sqrt{u-x}} d u
$$

has Mellin transform

$$
\left(D^{1 / 2} f\right)^{\star}(s)=-\frac{\Gamma(s)}{\Gamma(s-1 / 2)} f^{\star}(s-1 / 2),
$$

where $f^{\star}(s)$ is the usual Mellin transform of $f$. Hence by the Plancherel formula for the Mellin transform [38, Theorem 72]

$$
\begin{aligned}
\int_{0}^{\infty}\left(D^{1 / 2} f(x)\right)^{2} d x & =\frac{1}{2 \pi i} \int_{-i \infty}^{i \infty}\left(D^{1 / 2} f\right)^{\star}(s)\left(D^{1 / 2} f\right)^{\star}(1-s) d s \\
& =\frac{1}{2 \pi i} \int_{-i \infty}^{i \infty} \frac{\Gamma(s) \Gamma(1-s)}{\Gamma(s-1 / 2) \Gamma(1 / 2-s)} f^{\star}(s-1 / 2) f^{\star}(1 / 2-s) d s \\
& =\frac{1}{2 \pi} \int_{-\infty}^{\infty} f^{\star}(i y) f^{\star}(-i y) y \tanh (\pi y) d y ;
\end{aligned}
$$

also

$$
\int_{0}^{\infty}\left(D^{1 / 2} f(x)\right)^{2} d x=\frac{1}{4 \pi} \int_{-\infty}^{\infty}|\xi||\hat{f}(\xi)|^{2} d \xi=\int_{0}^{\infty} x C(f)(x)^{2} d x .
$$

Proposition 2.2. The following is a commuting diagram of linear isometries, in which the top arrow is the Fourier cosine transform, and the left downwards arrow is the Mellin transform.

$$
\begin{array}{cccc}
\left\{f: D^{1 / 2} f \in L^{2}(0, \infty)\right\} & \rightarrow & \left\{\phi \in L^{2}((0, \infty) ; x d x)\right\} \\
\downarrow & & \downarrow \\
\left\{f^{\star} \in L^{2}(i \mathbb{R} ; y \tanh (\pi y) d y /(2 \pi))\right\} & \rightarrow & \left\{\Gamma_{\phi} \in \mathcal{L}^{2}\right\}
\end{array}
$$

Proof. We have

$$
\left\|\Gamma_{\phi}\right\|_{\mathcal{L}^{2}}^{2}=\int_{0}^{\infty} \int_{0}^{\infty}|\phi(x+y)|^{2} d x d y=\int_{0}^{\infty} u|\phi(u)|^{2} d u,
$$

so the downwards map on the right is an isometry. The other maps are described in the preceding Lemma 2.1.

We show that trace class Hankel operators on Hardy space $H^{2}\left(\mathbb{C}_{+}\right)$have a matrix representation with respect to reproducing kernels on the state space. Let $\mathbb{C}_{+}=\{z \in \mathbb{C}: \operatorname{Re} z>0\}$ and $\mathbb{C}_{-}=\{z \in \mathbb{C}: \operatorname{Re} z<0\}$; then we introduce the usual Hardy spaces $H^{2}\left(\mathbb{C}_{+}\right)$and $H^{2}\left(\mathbb{C}_{-}\right)$which are related by the unitary involution $J: H^{2}\left(\mathbb{C}_{+}\right) \rightarrow$ $H^{2}\left(\mathbb{C}_{-}\right): f(s) \mapsto f(-s)$. We regard $H^{2}\left(\mathbb{C}_{+}\right)$as a closed linear subspace of $L^{2}(i \mathbb{R})$, and let $P_{+}: L^{2}(i \mathbb{R}) \rightarrow H^{2}\left(\mathbb{C}_{+}\right)$ be the orthogonal projection. For $h \in L^{\infty}(i \mathbb{R})$, let $M_{h}: L^{2}(i \mathbb{R}) \rightarrow L^{2}(i \mathbb{R})$ be the multiplication operator $f \mapsto h f$. The Laplace transform gives a unitary isometry $\mathcal{L}: L^{2}(0, \infty) \rightarrow H^{2}\left(\mathbb{C}_{+}\right)$. 
Given $c \in L^{\infty}$, suppose that $\Gamma_{c}=P_{+} M_{c} J$ is a bounded Hankel operator. Then by Nehari's and Fefferman's theorems [28], there exists $\psi \in L^{\infty}(i \mathbb{R})$ such that

$$
c(s)-c(\tau)=\int_{-\infty}^{\infty} \psi(i \omega)\left(\frac{1}{s-i \omega}-\frac{1}{\tau-i \omega}\right) \frac{d \omega}{2 \pi} \quad\left(s, \tau \in \mathbb{C}_{+}\right) .
$$

Note that $\psi$ determines $c$ up to an additive constant; adding a constant $\alpha$ to $c$ does not change $\psi$ or $\Gamma_{c}$. See [28].

Let $H=H^{2}\left(\mathbb{C}_{+}\right)$be the state space and let $\mathcal{D}(A)=\{g(s) \in H: s g(s) \in H\}$ with the graph norm. Then we introduce the linear system $(-A, B, C)$ by

$$
\begin{aligned}
A: \mathcal{D}(A) \rightarrow H: & g(s) \mapsto s g(s) \quad(g \in \mathcal{D}(A)) ; \\
B: \mathbb{C} \rightarrow \mathcal{D}(A)^{\star}: & \beta \mapsto \beta \quad(\beta \in \mathbb{C}) ; \\
C: \mathcal{D}(A) \rightarrow \mathbb{C}: & g \mapsto \frac{1}{2 \pi} \int_{-\infty}^{\infty} g(i \omega) \overline{c(i \omega)} d \omega \quad(g \in \mathcal{D}(A)) .
\end{aligned}
$$

The semigroup $\left(e^{-t A}\right)_{t>0}$ operates by multiplication on the state space and is strongly continuous, so $e^{-t A} f(s)=$ $e^{-s t} f(s)$. Let $k_{\zeta} \in H^{2}\left(\mathbb{C}_{+}\right)$be the function $k_{\zeta}(s)=1 /(s+\bar{\zeta})$, so that $\left\langle f, k_{\zeta}\right\rangle=f(\zeta)$ for all $f \in H^{2}\left(\mathbb{C}_{+}\right)$and $\zeta \in \mathbb{C}_{+}$; one calls $k_{\zeta}(s)$ the reproducing kernel of $H^{2}\left(\mathbb{C}_{+}\right)$. The various conjugates are introduced so that we can work with analytic, as opposed to anti-analytic, functions.

Lemma 2.3. Let $\operatorname{Re} \zeta_{j}>0$ and $c_{j} \in \mathbb{C}$ be such $\sum_{j=1}^{\infty}\left|c_{j}\right|\left|1+\zeta_{j}\right|^{2} / \operatorname{Re} \zeta_{j}$ is convergent.

(i) Then the series $c(s)=\sum_{j=1}^{\infty} c_{j} k_{\zeta_{j}}(s)$ converges in $H^{2}\left(\mathbb{C}_{+}\right)$and $H^{\infty}$;

(ii) the operators $\Gamma_{c}$ and $R_{x}=\int_{x}^{\infty} e^{-t A} B C e^{-t A} d t$ for $x>0$ are trace class on $H^{2}\left(\mathbb{C}_{+}\right)$;

(iii) $\Gamma_{c}^{\dagger}$ is unitarily equivalent to the Hankel integral operator $\Gamma_{\phi}$ on $L^{2}(0, \infty)$ with $\phi(t)=C e^{-t A} B$.

Proof. (i) The series $c(s)=\sum_{j=1}^{\infty} c_{j} k_{\zeta_{j}}(s)$ converges in $H^{2}\left(\mathbb{C}_{+}\right)$since $\sum_{j=1}^{\infty}\left|c_{j}\right| / \sqrt{\operatorname{Re} \zeta_{j}}$ converges. Also, $\|c\|_{L^{\infty}} \leq$ $\sum_{j=1}^{\infty}\left|c_{j}\right| / \operatorname{Re} \zeta_{j}<\infty$, so $c \in H^{\infty}\left(\mathbb{C}_{+}\right)$; hence we can choose $\psi(i \omega)=c(i \omega)$ in the above, and deduce that $(c(z)-c(\alpha)) /(\alpha-z)$ belongs to $H^{2}\left(\mathbb{C}_{+}\right)$with norm $m / \sqrt{\operatorname{Re} \alpha}$. Hence by Lemma 2.2 of [28], $C e^{-t A} g \in L^{2}(0, \infty)$ for all $g \in \mathcal{D}(A)$ with

$$
\int_{0}^{\infty}\left|C e^{-t A} g\right|^{2} d t \leq \frac{m\|g\|_{H}^{2}}{\operatorname{Re} \alpha} \quad(\operatorname{Re} \alpha>0, g \in \mathcal{D}(A)) .
$$

(iii) One can easily check that $e^{-t A^{*}} k_{\zeta}(s)=e^{-t \bar{\zeta}} k_{\zeta}(s)$, hence

$$
e^{-t A^{\star}} c(s)=\sum_{j=1}^{\infty} c_{j} e^{-t \bar{\zeta}_{j}} k_{\zeta_{j}}(s)
$$

We introduce

$$
\phi(t)=C e^{-t A} B=\left\langle 1, e^{-t A^{*}} c\right\rangle=\sum_{j=1}^{\infty} \bar{c}_{j} e^{-t \zeta_{j}} .
$$

From the expansion of $\phi(t+u)$ as a series of rank one kernels $e^{-\zeta_{j}(u+t)}$, we deduce that $\Gamma_{\phi}$ is trace class with $\left\|\Gamma_{\phi}\right\|_{\mathcal{L}^{1}} \leq \sum_{j}\left|c_{j}\right| /\left(2 \operatorname{Re} \zeta_{j}\right)$. One then checks that

$$
\left\langle\Gamma_{\phi}^{\dagger} f, g\right\rangle_{L^{2}(0, \infty)}=\left\langle\Gamma_{c} \mathcal{L} f, \mathcal{L} g\right\rangle_{H^{2}}
$$

the simplest way to do this is by selecting $f(x)=e^{-\xi x}$ and $g(x)=e^{-\zeta x}$, so that

$$
\left\langle\Gamma_{\phi}^{\dagger} f, g\right\rangle_{L^{2}(0, \infty)}=\sum_{j=1}^{\infty} \frac{c_{j}}{\left(\bar{\zeta}_{j}+\xi\right)\left(\bar{\xi}+\bar{\zeta}_{j}\right)}=\left\langle\frac{c(s)-c(\xi)}{\xi-s}, \frac{1}{\zeta+s}\right\rangle_{H^{2}} .
$$


Also, we deduce that

$$
\begin{gathered}
\psi(i \xi)=\int_{0}^{\infty} e^{-i \xi t} \bar{\phi}(t) d t, \\
\bar{\phi}(t)=\int_{-\infty}^{\infty} \psi(i \xi) e^{i \xi t} \frac{d \xi}{2 \pi} \quad(t>0) .
\end{gathered}
$$

(ii) Hence we can write

$$
\begin{aligned}
R_{X} f(z) & =\int_{x}^{\infty} e^{-t A} B C e^{-t A} f(z) d t \\
& =\sum_{j=1}^{\infty} \frac{\bar{c}_{j} e^{-x\left(z+\zeta_{j}\right)} f\left(\zeta_{j}\right)}{z+\zeta_{j}} \\
& =\sum_{j=1}^{\infty} \bar{c}_{j} e^{-x A} k_{\bar{\zeta}_{j}}(z)\left\langle f, e^{-x A^{\star}} k_{\zeta_{j}}\right\rangle
\end{aligned}
$$

so $R_{X} \in \mathcal{L}^{1}(H)$. Hence $\Gamma_{\phi}$ and $\Gamma_{c}$ are trace class operators.

Alternatively, one can introduce the sequence of $\lambda_{j}=\left(1-\zeta_{j}\right) /\left(1+\zeta_{j}\right)$ which satisfies $\left|\lambda_{j}\right|<1$ and $\sum_{j=1}^{\infty}\left|c_{j}\right| /\left(1-\left|\lambda_{j}\right|\right)<\infty$. Then one can show that $\Gamma_{c}$ is unitarily equivalent to a trace-class Hankel operator on the Hardy space $H^{2}$ of the unit disc, by Peller's criterion [29, p. 232]. Incidentally, Peller's criterion is sharp.

Any bounded Hankel integral operator generates a sequence of moments, in the following sense. For $\phi \in$ $L^{2}(0, \infty)$, let $\Gamma_{\phi}$ be the Hankel integral operator and introduce the moment sequence

$$
\begin{aligned}
\mu_{n} & =\int_{0}^{\infty} \phi(x) L_{n}(x) e^{-x / 2} d x \\
& =\frac{1}{2 \pi} \int_{-\infty}^{\infty} \hat{\phi}(\xi) \frac{(i \xi-1 / 2)^{n}}{(i \xi+1 / 2)^{n+1}} d \xi \\
& =\frac{1}{2 \pi i} \int_{|z|=1} \hat{\phi}\left(\frac{1+z}{2 i(1-z)}\right) z^{n} \frac{d z}{1-z} \quad(n=0,1, \ldots) .
\end{aligned}
$$

Magnus has characterized the moment sequences that arise as $\left(\mu_{n}=\int_{S} x^{n} w(x) d x\right)$ for a semi classical weight on some subset of $\mathbb{C} \cup\{\infty\}$, as we discuss in the next section.

\section{From orthogonal polynomials to Hankel determinants}

Let $\left(p_{n}(x)\right)_{n=0}^{\infty}$ be the sequence of monic orthogonal polynomials of degree $n$ for some continuous and positive weight $w_{0}(x)$ on $(0, b)$, given by the recurrence relation

$$
x p_{n}(x)=p_{n+1}(x)+\alpha_{n} p_{n}(x)+\beta_{n} p_{n-1}(x) .
$$

Let $\int p_{n}(x)^{2} w_{0}(x) d x=h_{n}$; then $\beta_{n}=h_{n} / h_{n-1}>0$. Let $Q_{n}$ be the orthogonal projection of $L^{2}(0, b)$ onto

$$
\operatorname{span}\left\{\sqrt{w_{0}(x)} p_{j}(x) ; j=0, \ldots, n-1\right\} .
$$

Then the Christoffel-Darboux formula gives

$$
Q_{n}(x, y)=\frac{1}{h_{n-1}} \sqrt{w_{0}(x) w_{0}(y)} \frac{p_{n}(x) p_{n-1}(y)-p_{n}(y) p_{n-1}(x)}{x-y}
$$


so that $Q_{n}$ is an integrable operator. We show also that for suitable weights, $Q_{n}$ is a sum of products of Hankel operators.

Definition 3.1. (Magnus, [24]) (i) Let $F(z)=\int_{0}^{b}(z-x)^{-1} w_{0}(x) d x$ be the Cauchy transform of the weight $w_{0}$ on $E=(0, b)$. The weight is said to be semi-classical if there exist polynomials $U, V, W$ with $W \neq 0$ such that

$$
W(z) F^{\prime}(z)=2 V(z) F(z)+U(z) \quad(z \in \mathbb{C} \backslash \mathbb{R}) .
$$

Equivalently, the moments $\mu_{k}=\int x^{k} w_{0}(x) d x$ satisfy a recurrence relation

$$
\sum_{j=0}^{m}\left(v \xi_{j}+\eta_{j}\right) \mu_{j+v}=0 \quad(v=0,1 \ldots),
$$

for some $\xi_{j}, \eta_{k} \in \mathbb{C}$ given by the coefficients of $V, W$, where $m$ is the maximum of the degrees of the polynomials $V$ and $W$.

(ii) A pair of polynomials $(2 V, W)$ is said to be generic if $W$ has degree $m$ where $m \geq 2$, the degree of $V$ is less than $m, W$ has $m$ simple zeros $\alpha_{j}$ and $2 V / W$ has all residues $2 V\left(\alpha_{j}\right) / W^{\prime}\left(\alpha_{j}\right)$ that are not integers.

(iii) Let $\vartheta$ be Heaviside's function, so $\vartheta(x)=0$ for $x<0$ and $\vartheta(x)=1$ for $x \geq 0$.

Theorem 3.2. Let $w_{0}$ be a positive and continuous semi-classical weight on $[0, \infty)$ that corresponds to a generic pair $(2 \mathrm{~V}, W)$, and let (1.2) be the corresponding probability measure.

(i) Then there exist $\phi_{j}, \psi_{j} \in L^{2}(0, \infty)$ such that

$$
Q_{n}(x, y)=\sum_{j=1}^{N} \int_{0}^{\infty} \phi_{j}(x+t) \psi_{j}(t+y) d t .
$$

(ii) There exist scattering functions $\Phi, \Psi \in L^{2}\left((0, \infty)\right.$; $\left.\mathbb{C}^{N}\right)$ such that, for all $f \in L^{\infty}(\mathbb{R})$ as in (1.3),

$$
\mathbb{E} e^{-\sum f\left(x_{j}\right)}=\operatorname{det}\left(I-M_{h} \Gamma_{\Psi}^{T} \Gamma_{\Phi}\right),
$$

where $h=1-e^{-f}$ and ${ }^{T}$ denotes transpose.

(iii) For $f(x)=\beta 9(x-t)$ with $\operatorname{Re} \beta>0$ and $\lambda=1-e^{-\beta}$, the moment generating function of the random variable $\sharp\left\{j: x_{j}>t\right\}$ subject to the probability (1.2) is given by

$$
\mathbb{E} e^{-\sum \vartheta\left(x_{j}-t\right)}=\operatorname{det}\left(I-\lambda \Gamma_{\Psi_{t}}^{T} \Gamma_{\Phi_{t}}\right),
$$

where the scattering functions are shifted to $\Phi_{t}(x)=\Phi(x+t)$ and $\Psi_{t}(x)=\Psi(x+t)$.

Proof. (i) Magnus [24] shows that for each such polynomial pair, there exists a weight $w_{0}$ with Cauchy transform $F$ and a polynomial $U$ such that $W F^{\prime}=2 V F+U$. From [24, (11)], we have $W w_{0}^{\prime}=2 V w_{0}$. Then by [24, (17)], there exist polynomials $\Omega_{n}$ and $\Theta_{n}$, and recursion coefficients $a_{n}$ such that with the matrices

$$
Y_{n}(x)=\left[\begin{array}{c}
\sqrt{w_{0}(x)} p_{n}(x) \\
\sqrt{w_{0}(x)} p_{n-1}(x)
\end{array}\right] ; \quad J=\left[\begin{array}{cc}
0 & -1 \\
1 & 0
\end{array}\right] ; \quad A_{n}(x)=\frac{1}{W(x)}\left[\begin{array}{cc}
\Omega_{n}(x) & -a_{n} \Theta_{n}(x) \\
a_{n} \Theta_{n-1}(x) & -\Omega_{n}(x)
\end{array}\right]
$$

we have an ordinary differential equation

$$
\frac{d}{d x} Y_{n}(x)=A_{n}(x) Y_{n}(x),
$$

where the coefficient matrix $A_{n}(x)$ is rational with trace equal to zero. The three-term recurrence relation (3.1) for $p_{n}$ gives a positive sequence $\left(\beta_{n}\right)$ and a real sequence $\left(\alpha_{n}\right)$ such that

$$
Y_{n+1}=\left[\begin{array}{cc}
x-\alpha_{n} & -\beta_{n} \\
1 & 0
\end{array}\right] Y_{n}
$$


so we have a recurrence relation for the matrices in (3.9)

$$
A_{n+1}\left[\begin{array}{cc}
x-\alpha_{n} & -\beta_{n} \\
1 & 0
\end{array}\right]=\left[\begin{array}{cc}
x-\alpha_{n} & -\beta_{n} \\
1 & 0
\end{array}\right] A_{n}+\left[\begin{array}{ll}
1 & 0 \\
0 & 0
\end{array}\right],
$$

where the second matrix has determinant $\beta_{n}>0$, hence $\left(A_{n}\right)_{n=0}^{\infty}$ is uniquely determined by the data $A_{0},\left(\alpha_{n}\right)_{n=0}^{\infty}$ and $\left(\beta_{n}\right)_{n=0}^{\infty}$. This is the matrix form of the Freud equation for orthogonal polynomials, otherwise known as the discrete string equation for the weight $w_{0}$ [8, p. 989]. We can therefore follow the approach of [40, section VI]. From the differential equation (3.9),

$$
\begin{aligned}
\left(\frac{\partial}{\partial x}+\frac{\partial}{\partial y}\right) Q_{n}(x, y) & =\frac{1}{h_{n-1}}\left(\frac{\partial}{\partial x}+\frac{\partial}{\partial y}\right) \frac{\left\langle J Y_{n}(x), Y_{n}(y)\right\rangle}{x-y} \\
& =\frac{1}{h_{n-1}} \frac{\left\langle J A_{n}(x) Y_{n}(x), Y_{n}(y)\right\rangle+\left\langle J Y_{n}(x), A_{n}(y) Y_{n}(y)\right\rangle}{x-y} \\
& =\frac{1}{h_{n-1}}\left\langle B_{n}(x, y) Y_{n}(x), Y_{n}(y)\right\rangle,
\end{aligned}
$$

where $B_{n}(x, y)=J A_{n}(x)+A_{n}(y)^{T} J$ is given explicitly by

$$
B_{n}(x, y)=\left[\begin{array}{cc}
\frac{\left(a_{n} \Theta_{n-1} / W\right)(y)-\left(a_{n} \Theta_{n-1} / W\right)(x)}{x-y} & \frac{\left(\Omega_{n} / W\right)(x)-\left(\Omega_{n} / W\right)(y)}{x-y} \\
\frac{\left(\Omega_{n} / W\right)(x)-\left(\Omega_{n} / W\right)(y)}{x-y} & \frac{\left(a_{n} \Theta_{n} / W\right)(y)-\left(a_{n} \Theta_{n} / W\right)(x)}{x-y}
\end{array}\right],
$$

which is rational, symmetric with respect to interchange of variables $x \leftrightarrow y$ and symmetric with respect to matrix transpose. Using the identity $W w_{0}^{\prime}=2 w_{0} V$, and canceling any common zeros of $V$ and $W$, we deduce that $W$ has no zeros on $(0, \infty)$, since $w_{0}(x)>0$ for all $x>0$ by hypothesis. Observe also that $\int_{0}^{\infty} x^{k} w_{0}(x) d x$ is finite for all $k \in \mathbb{N} \cup\{0\}$. By selecting the products of functions that depend on one variable, namely $x$ or $y$, we can therefore choose $\phi_{j}$ and $\phi_{k}$ from among the functions in $B$ and $Y$ such that $\phi_{j}, \psi_{j} \in L^{2}(0, \infty)$ and

$$
\left(\frac{\partial}{\partial x}+\frac{\partial}{\partial y}\right) Q_{n}(x, y)=-\sum_{j=1}^{N} \phi_{j}(x) \psi_{j}(y)
$$

By integration, we obtain

$$
Q_{n}(x, y)=\sum_{j=1}^{N} \int_{0}^{\infty} \phi_{j}(x+t) \psi_{j}(t+y) d t+q(x-y)
$$

where $q(x-y) \rightarrow 0$ as $x \rightarrow \infty$ or $y \rightarrow \infty$, so $q=0$. We can select the $\phi_{j}, \psi_{j}$ so that $\int_{0}^{\infty} x\left|\phi_{j}(x)\right|^{2} d x$ and $\int_{0}^{\infty} x\left|\psi_{j}(x)\right|^{2} d x$ are all finite, so $\Gamma_{\phi_{j}}$ and $\Gamma_{\psi_{j}}$ are Hilbert-Schmidt.

(ii) Let $h(x)=1-e^{-f(x)}$ for some $f \in L^{\infty}$, so that $e^{-\sum_{j=1}^{n} f\left(x_{j}\right)}=\prod_{j=1}^{n}\left(1-h\left(x_{j}\right)\right)$. Then with the probability measure (1.2), the expectation of this product is

$$
\begin{aligned}
\mathbb{E} e^{-\sum f} & =\frac{\int_{(0, \infty)^{n}} \prod_{1 \leq j<k \leq n}\left(x_{j}-x_{k}\right)^{2} \prod_{j=1}^{n}\left(1-h\left(x_{j}\right)\right) w_{0}\left(x_{j}\right) d x_{j}}{\int_{(0, \infty)^{n}} \prod_{1 \leq j<k \leq n}\left(x_{j}-x_{k}\right)^{2} \prod_{j=1}^{n} w_{0}\left(x_{j}\right) d x_{j}} \\
& =\operatorname{det}\left(I-M_{h} Q_{n}\right) .
\end{aligned}
$$

We let $\Phi:(0, \infty) \rightarrow \mathbb{C}^{N \times 1}$ be $\Phi(x)=\operatorname{column}\left[\phi_{j}(x)\right]_{j=1}^{N}$ and $\Psi:(0, \infty) \rightarrow \mathbb{C}^{N \times 1}$ be

$$
\Psi(x)=\operatorname{column}\left[\psi_{j}(x)\right]_{j=1}^{N},
$$

as in (3.15), so

$$
\begin{aligned}
\mathbb{E} e^{-f} & =\operatorname{det}\left(I-M_{h} \Gamma_{\Phi}^{T} \Gamma_{\Psi}\right) \\
& =\operatorname{det}\left(I-\Gamma_{\Psi} M_{h} \Gamma_{\Phi}^{T}\right),
\end{aligned}
$$


where the final operator has a matrix kernel

$$
\Gamma_{\Psi} M_{h} \Gamma_{\Phi}^{T} \leftrightarrow\left[\int_{0}^{\infty} \psi_{j}(x+u)\left(1-e^{-f(u)}\right) \phi_{k}(u+y) d u\right]_{j, k=1}^{N} .
$$

(iii) For $\operatorname{Re} \beta>0$, the point $\lambda=1-e^{-\beta}$ lies in the disc of centre 1 and radius 1 in $\mathbb{C}$. Then for the step function $f(x)=\beta 9(x-t)$ we have

$$
\begin{aligned}
\mathbb{E} e^{-\beta \sum \vartheta(.-t)} & =\sum_{k=0}^{\infty} e^{-k \beta} \mathbb{P}\left[\natural\left\{j: x_{j} \in(t, \infty)\right\}=k\right] \\
& =\sum_{k=0}^{\infty}(1-\lambda)^{k} \mathbb{P}\left[\natural\left\{j: x_{j} \in(t, \infty)\right\}=k\right] \\
& =\sum_{k=0}^{\infty} \frac{(1-\lambda)^{k}}{k !}\left(\frac{d^{k}}{d \mu^{k}}\right)_{\mu=1} \operatorname{det}\left(I-\mu M_{\vartheta(.-t)} Q_{n}\right),
\end{aligned}
$$

so we have the moment generating function of the number of the $x_{j}$ that are greater than $t$. Then

$$
\left(1-e^{-\beta}\right) \Gamma_{\Psi} M_{\vartheta(.-t)} \Gamma_{\Phi}^{T} \leftrightarrow \lambda\left[\int_{0}^{\infty} \psi_{j}(x+u+t) \phi_{k}(u+t+y) d u\right]_{j, k=1}^{N},
$$

where each entry of the matrix is a product of Hankel operators, with scattering functions $\psi_{j}(x)$ and $\phi_{k}(x)$ shifted to $\psi_{j}(x+t)$ and $\phi_{k}(x+t)$.

Theorem 3.2 involves a Fredholm determinant. The following result gives an equivalent expression involving finite determinants on the numerator. We introduce the block matrix

$$
\Theta_{t}=\left[\begin{array}{ccc}
0_{N \times N} & \Psi_{t} & 0_{N \times(N-1)} \\
\Phi_{t}^{T} & 0 & 0_{1 \times(N-1)} \\
0_{(N-1) \times N} & 0_{(N-1) \times 1} & 0_{(N-1) \times(N-1)}
\end{array}\right] .
$$

Corollary 3.3. Suppose that $\Gamma_{\Theta_{t}} \in \mathcal{L}^{1}$ and $I+\sqrt{\lambda} \Gamma_{\Theta_{t}}$ is invertible.

(i) Then for any orthogonal projection $P_{n}$ on $L^{2}\left((0, \infty) ; \mathbb{C}^{2 N}\right)$ with $P_{n}^{\perp}=I-P_{n}$,

$$
\mathbb{E} e^{-\beta \sum \vartheta(.-t)}=\frac{\operatorname{det} P_{n}\left(I+\sqrt{\lambda} \Gamma_{\Theta_{t}}\right) P_{n}}{\operatorname{det} P_{n}^{\perp}\left(I+\sqrt{\lambda} \Gamma_{\Theta_{t}}\right)^{-1} P_{n}^{\perp}} .
$$

(ii) Let $L_{j}(x)$ be the Laguerre polynomial, and let $P_{n}$ be the orthogonal projection onto

$$
\operatorname{span}\left\{e^{-x / 2} L_{j}(x): j=0, \ldots, n\right\} \otimes \mathbb{C}^{2 N} .
$$

Then $P_{n} \Gamma_{\Theta_{t}} P_{n}$ is unitarily equivalent to a finite block Hankel matrix.

Proof. (i) We have especially chosen $\Theta$ so that by Theorem 3.2(iii), we have

$$
\mathbb{E} e^{-\beta \sum \vartheta(.-t)}=\operatorname{det}\left(I+\sqrt{\lambda} \Gamma_{\Theta_{t}}\right) .
$$

Then the stated result follows from a determinant formula credited to Jacobi; see [2].

(ii) Hankel integral operators correspond to Hankel matrices via the Laguerre orthonormal basis of $L^{2}(0, \infty)$; see [29, p. 53]. (This is a special feature of the Laguerre polynomials.) To extend this to Hankel integral operators on $L^{2}\left((0, \infty) ; \mathbb{C}^{2 N}\right)$, we just compute the block Hankel matrix

$$
\left[\int_{0}^{\infty} \Theta_{t}(x) e^{-x / 2} L_{j+k}(x) d x\right]_{j, k=0}^{n-1}
$$

which has $(2 N) \times(2 N)$ block entries, and has the cross-diagonal pattern that is characteristic of Hankel matrices. 
Theorem 3.2 shows that replacing $w(x)$ by $w(x) e^{-\beta 9(x-t)}$ corresponds shifting $\Theta_{0}$ to $\Theta_{t}$. The shift operation is simple to describe in terms of linear systems, as in (5.12). Unfortunately, $\vartheta$ is discontinuous, so $w(x) e^{-\beta \vartheta(x-t)}$ is not itself a semiclassical weight, and we cannot immediately deduce a differential equation such as (3.9) for orthogonal polynomials generated by $w(x) e^{-\beta \vartheta(x-t)}$. In the following results, we introduce a family of semiclassical weights involving

$$
f(x)=\beta \tan ^{-1} \frac{x-t}{\varepsilon}
$$

for $\varepsilon>0$, and $t, \beta \in \mathbb{R}$, since $f(x) \rightarrow \beta \pi(\vartheta(x-t)-1 / 2)$ as $\varepsilon \rightarrow 0+$. The motivation is that the family of $f$ approximates the $\beta \pi(\vartheta(x-t)-1 / 2)$ as $\varepsilon \rightarrow 0+$, and gives a family of rational linear differential equations with deformation parameter $\varepsilon$, to which we can apply Schlesinger's theory of isomonodromic deformations to obtain information about the Hankel determinants.

As in Theorem 3.2, we suppose that $w_{0}$ satisfies $W w_{0}^{\prime}=2 V w_{0}$, where $V, W$ are polynomials, and let $v_{0}=-\log w_{0}$. Then there exists $\varepsilon_{0}>0$ such that

$$
\left(2 V(x)\left(x-z_{+}\right)\left(x-z_{-}\right)+i \beta\left(z_{+}-z_{-}\right) W(x), W(x)\left(x-z_{+}\right)\left(x-z_{-}\right)\right)
$$

is also generic for all real $\beta$ and $0<\operatorname{Im} z_{+}<\varepsilon_{0}$ and $0<-\operatorname{Im} z_{-}<\varepsilon_{0}$. In particular, we can replace our previous weight $w_{0}(x)$ by

$$
w(x)=w_{0}(z)\left(x-z_{+}\right)^{i \beta / 2}\left(x-z_{-}\right)^{-i \beta / 2}
$$

then we build the system of monic orthogonal polynomials $\left(p_{j}(x)\right)_{j=0}^{\infty}$ for the complex bilinear form $\langle f, g\rangle=$ $\int_{E} f(x) g(x) w(x) d x$.

Proposition 3.4. Suppose that $(2 \mathrm{~V}, W)$ is generic.

(i) Then there exists $\varepsilon_{0}>0$ such that

$$
\left(2 V(x)\left(\varepsilon^{2}+(x-t)^{2}\right)+\varepsilon \beta W(x), W(x)\left(\varepsilon^{2}+(x-t)^{2}\right)\right),
$$

is generic for all real $\beta$ and $0<\varepsilon<\varepsilon_{0}$;

(ii) there exists a consistent system of ordinary differential equations as in (3.8) and (3.9)

$$
\begin{aligned}
& \frac{d Y}{d x}=A(x, t ; \beta, \varepsilon) Y \\
& \frac{d Y}{d t}=H(x, t ; \beta, \varepsilon) Y,
\end{aligned}
$$

where $A(x, t ; \beta, \varepsilon)$ is a proper rational function of $x$ with trace zero, and simple poles at the zeros of $W$ and $t \mp i \varepsilon$;

(iii) the consistency condition holds

$$
\frac{\partial A}{\partial t}-\frac{\partial H}{\partial x}+[A, H]=0
$$

Proof. (i) This is a direct check of the definitions. Then the modified potential $v=-\log w$ has $v^{\prime}$ rational, and we obtain a family of pairs of polynomials, depending upon parameters $(t, \varepsilon, \beta)$. For given $n$, we can choose $\varepsilon_{0}>0$ such that the Gram-Schmidt process for the bilinear form $\langle f, g\rangle$ produces orthogonal polynomials of degree up to $n$, for all $0<\varepsilon<\varepsilon_{0}$.

(ii) Magnus [24] obtains $\Theta_{n}$ and $\Omega_{n}$ by recursion, and one checks that the degree of $\Theta_{n}$ is less than or equal to $m$, while the degree of the denominator is $m+2$. From his recursion formula [24, (20)], the degree of $\Omega_{n}^{2}$ is less than or equal to $2(m+1)$, so $A(x, t ; \beta, \varepsilon)$ is strictly proper. By (3.9) and Proposition 3.4(i), we can write

$$
A\left(x, z_{ \pm}\right)=\frac{A_{+}}{x-z_{+}}+\frac{A_{-}}{x-z_{-}}+\sum_{j=1}^{m} \frac{A_{j}}{x-\alpha_{j}},
$$

where the $2 \times 2$ residue matrices $A_{j}, A_{ \pm}$depend upon $\left(\beta, z_{ \pm}\right)$, but not upon $x$. The set of singular points in the Riemann sphere $\mathbb{C} \cup\{\infty\}$ is $\left\{\alpha_{1}, \ldots, \alpha_{m}, z_{ \pm}, \infty\right\}$. 
We can take $z_{ \pm}=t \pm i \varepsilon$, a complex conjugate pair. Then we fix $\beta \in \mathbb{R}$ and some $0<\varepsilon<\varepsilon_{0}$ and regard $t$ as the main deformation parameter. Then the weight

$$
w(x)=w_{0}(x) \exp \left(\frac{\beta \pi}{2}-\beta \tan ^{-1} \frac{x-t}{\varepsilon}\right)
$$

is positive and continuous on $E$, so $p_{j}$ is a real polynomial and $h_{j}>0$. Since the differential equation (3.27) has only regular singular points, the monodromy is fully described in [30] by results of Schlesinger [30, p. 148] and Dekkers [30, p. 180] in terms of connections of dimension two on the punctured Riemann sphere. Schlesinger found the condition for the system to undergo an infinitesimal change in the poles $\left\{\alpha_{1}, \ldots, \alpha_{m} ; z_{ \pm}\right\}$that does not change the monodromy. Let $Y$ be the fundamental solution matrix of (3.27), and introduce

$$
\begin{aligned}
H & =\frac{\partial Y}{\partial t} Y^{-1} \\
& =\left(\frac{\partial Y}{\partial z_{+}}+\frac{\partial Y}{\partial z_{-}}\right) Y^{-1} \\
& =-\frac{A_{+}}{x-z_{+}}-\frac{A_{-}}{x-z_{-}}
\end{aligned}
$$

to obtain the required variation in $z_{\mp}$.

(iii) This formula follows from the equality of mixed partial derivatives $\partial^{2} Y / \partial t \partial x=\partial^{2} Y / \partial x \partial t$ where $Y$ is the fundamental solution matrix of (3.27) and $\partial / \partial t=\partial / \partial z_{+}+\partial / \partial z_{-}$. To ensure that the differential equations are indeed consistent, we require

$$
\frac{\partial A\left(x, z_{ \pm}\right)}{\partial t}=\frac{A_{+}}{\left(x-z_{+}\right)^{2}}+\frac{A_{-}}{\left(x-z_{-}\right)^{2}}+\frac{\frac{\partial A_{+}}{\partial t}}{x-z_{+}}+\frac{\frac{\partial A_{-}}{\partial t}}{x-z_{-}}+\sum_{j=1}^{m} \frac{\frac{\partial A_{j}}{\partial t}}{x-\alpha_{j}},
$$

where by Schlesinger's equations

$$
\begin{gathered}
\frac{\partial A_{ \pm}}{\partial t}=-\sum_{j=1}^{m} \frac{\left[A_{j}, A_{ \pm}\right]}{\alpha_{j}-z_{ \pm}} \\
\frac{\partial A_{j}}{\partial t}=\frac{\left[A_{j}, A_{+}\right]}{\alpha_{j}-z_{+}}+\frac{\left[A_{j}, A_{-}\right]}{\alpha_{j}-z_{-}} \quad(j=1, \ldots, m) .
\end{gathered}
$$

Corollary 3.5. Suppose in (3.30) that $m=1$, that $A_{+}+A_{-}+A_{1}$ is a diagonal matrix and

$$
\operatorname{trace} A_{+}=\operatorname{trace} A_{-}=\text {trace } A_{1}=0 .
$$

Then (3.29) reduces to a Painlevé VI equation.

Proof. By translating $z$ to $z+t$, we replace the singular points $\left(t-i \varepsilon, t+i \varepsilon, \alpha_{1}, \infty\right)$ by $\left(-i \varepsilon,+i \varepsilon, \alpha_{1}-t, \infty\right)$, so we have variation in only one pole. Then we can apply known results from [17] and [19] to reduce the compatibility condition (3.29) to a Painlevé VI ordinary differential equation.

Remark 3.6. (i) By taking $\varepsilon \rightarrow 0+$, have $z_{ \pm} \rightarrow t$ and

$$
D_{n}[w]=\operatorname{det}\left[\int_{0}^{\infty} x^{j+k} e^{2 \pi \beta(1-\vartheta(x-t))} w_{0}(x) d x\right]_{j, k=0}^{n-1} .
$$

In section 8 , we consider the equilibrium problem for this determinant for large $n$.

(ii) Chen and Its [8] showed that the Hankel determinant $D[w]$ gives the isomonodromic $\tau$ function for the system of Schlesinger equations that describe the isomonodromic deformation of (3.27) with respect to the position of the poles. The Schlesinger equations may be solved in terms of the $\Theta$-function on a hyperelliptic Riemann surface, as in [22]. The solutions to the monodromy preserving differential equations have singularities which are poles, except for the fixed singularities. Previously, Magnus [24] had found conditions for the 
system (3.8) to undergo an isomonodromic deformation, and obtained examples that realize the nonlinear Painlevé VI equation as (3.29). Min Chao and Chen [26] derived an ODE for gap probabilities in the Jacobi ensemble

(iii) Tracy and Widom considered Fredholm determinants $\operatorname{det}\left(I-\Gamma_{\phi_{t}}^{2}\right)$ for classical orthogonal polynomials [39], [40] and computed $(d / d t) \log \operatorname{det}\left(I-\Gamma_{\Phi_{t}} \Gamma_{\Psi_{t}}\right)$ in terms of operator kernels. They identified weights that produce Painlevé $I I, I I I, I V$ and $V$. For differential equations (3.9) with $W(x)=1$, that have polynomial coefficients, Palmer [27] identified $\operatorname{det}\left(I-\Gamma_{\phi_{t}}^{2}\right)$ as the $\tau$-function of the ODE (3.9) for isomonodromic deformations. His analysis addressed the case in which infinity is an irregular singular point.

\section{Wiener-Hopf Factorization}

In section 4, we saw how Hankel products arise from the differential equations (3.9) and (3.14). In this section we show how they arise from the Wiener-Hopf factorization. Both of these routes are familiar in the theory of random matrices.

Fix $0<\varepsilon<1$. Let $\mathcal{C}_{2}^{0}=\mathcal{C}_{2}^{0}(\varepsilon)$ be the space of functions $f$ such that:

(i) $f$ is bounded and analytic on the strip $S_{\varepsilon}=\{z:|\operatorname{Re} z|<\varepsilon\}$;

(ii) $f(\eta+i \xi) \rightarrow 0$ as $\xi \rightarrow \pm \infty$, uniformly for $|\eta| \leq \varepsilon / 2$;

(iii)

$$
\sup _{|\eta|<\varepsilon / 2} \int_{-\infty}^{\infty}|f(\eta+i \xi)|^{2} d \xi<\infty
$$

Let $\mathcal{C}_{2}=\mathcal{C}_{2}^{0}+\mathbb{C}$.

Proposition 4.1. (i) Then $\mathrm{C}_{2}$ is a commutative and unital Banach algebra under the usual pointwise multiplication.

(ii) Let $f \in \mathcal{C}_{2}^{0}$ with $U=\left\{f(z): z \in S_{\varepsilon}\right\}$, let $V$ be a neighbourhood of the closure of $U$ and let $\varphi: V: \rightarrow \mathbb{C}$ be a holomorphic function such that $\varphi(0)=0$. Then $\varphi(f) \in \mathcal{C}_{2}^{0}$.

(ii) There is a bounded linear map $\psi \mapsto \Gamma_{\phi}^{\dagger}$ from $\mathrm{C}_{2}^{0} \rightarrow \mathcal{L}^{2}$ via the transform (2.17).

Proof. (i) We take the norm to be

$$
\|f\|_{\mathrm{e}_{2}}=\sup _{z}\{|f(z)|: z=x+i y ; y \in \mathbb{R}, x \in(-\varepsilon, \varepsilon)\}+\sup _{-\varepsilon / 2<x<\varepsilon / 2}\left(\int_{-\infty}^{\infty}|f(x+i y)|^{2} d y\right)^{1 / 2} .
$$

Evidently $\mathcal{C}_{2}$ is a subspace of the Banach algebra $H^{\infty}$ of bounded functions on the strip $S_{\varepsilon}=\{z:|\operatorname{Re} z|<\varepsilon\}$, hence $\mathcal{C}_{2}$ is an integral domain.

(ii) We can choose a contour $\Gamma$ in $V \backslash U$ that winds once round $U$ in the positive sense, so that

$$
\varphi(f)=\frac{f}{2 \pi i} \int_{\Gamma} \frac{\varphi(\lambda) d \lambda}{(\lambda-f) \lambda}
$$

holds by Cauchy's theorem, where the right-hand side is evidently in $\mathcal{C}_{2}^{0}$. 
(iii) The Hankel integral operator with kernel $\phi(s+t)$ on $L^{2}(0, \infty)$ has Hilbert-Schmidt norm satisfying

$$
\begin{aligned}
\|\Gamma(\phi)\|_{\mathcal{L}^{2}}^{2} & =\int_{0}^{\infty} t|\phi(t)|^{2} d t \\
& \leq \int_{0}^{\infty}\left(1+t^{2}\right)|\phi(t)|^{2} d t \\
& =\int_{-\infty}^{\infty}|\psi(i \xi)|^{2} d \xi+\int_{-\infty}^{\infty}\left|\psi^{\prime}(i \xi)\right|^{2} d \xi,
\end{aligned}
$$

where we have used Plancherel's formula. By Cauchy's integral formula for derivatives, we have

$$
\begin{aligned}
\int_{-\infty}^{\infty}\left|\psi^{\prime}(i \xi)\right|^{2} d \xi & \leq \frac{1}{\pi \varepsilon} \int_{0}^{2 \pi} \int_{-\infty}^{\infty}\left|\psi\left(i \xi+\varepsilon e^{i \theta} / 2\right)\right|^{2} d \xi d \theta \\
& \leq \frac{2}{\varepsilon} \sup _{|\eta|<\varepsilon / 2} \int_{-\infty}^{\infty}|\psi(i \xi+\eta)|^{2} d \xi
\end{aligned}
$$

Hence $\Gamma(\check{f})$ is a Hilbert-Schmidt operator.

By composing the transformations $z \mapsto(z+\varepsilon) /(4 \varepsilon)$ followed by $z \mapsto(z-1) /(z+1)$, we map $S_{\varepsilon}$ to the region bounded by the circles $C(0,1)$ and $C(1 / 3,2 / 3)$, which has a closure that is shaped like an earring and is not simply connected. Consider the multiplicative group $G\left(\mathcal{C}_{2}\right)=\left\{f \in \mathcal{C}_{2}: \exists g \in \mathcal{C}_{2}, f g=1\right\}$ with subgroup $\exp \left(\mathcal{C}_{2}\right)=\left\{\exp (f): f \in \mathcal{C}_{2}\right\}$. Employing more classical language, Titchmarsh [38] identified a subgroup of $G\left(\mathcal{C}_{2}\right) / \exp \left(\mathcal{C}_{2}\right)$ with $\mathbb{Z}$. Let $\psi$ be typical element of $\mathcal{C}_{2}$ such that $\psi(z) \rightarrow 1$ as $z \rightarrow \pm \infty$ along the imaginary axis and such that $\psi$ has no zeros on the imaginary axis. The function $|\log \psi(\eta+i \xi)|$ is square integrable for $-\varepsilon / 2 \leq \eta \leq \varepsilon / 2$. Then $\psi$ has the form

$$
\psi(z)=\left(\frac{z-1}{z+1}\right)^{k} \frac{\prod_{j=1}^{m}\left(z-w_{j}\right)}{\left(z^{2}-1\right)^{m / 2}} \exp \left(\chi_{+}(z)-\chi_{-}(z)\right)
$$

where (1) $w_{j}$ are the zeros of $\psi(z)$ for $|\operatorname{Re} z| \leq \varepsilon / 2$,

(2) $k$ is the winding number of the contour $\{\psi(i \xi):-\infty \leq \xi \leq \infty\}$,

(3) $\chi_{+}$is holomorphic and bounded on $\operatorname{Re} z \geq-\varepsilon / 2$ and

$$
\chi_{+}(w)=\frac{1}{2 \pi i} \int_{-i \infty-\varepsilon / 2}^{i \infty-\varepsilon / 2} \frac{\log \psi(z)}{z-w} d z,
$$

(4) $\chi$ - is holomorphic and bounded on $\operatorname{Re} z \leq \varepsilon / 2$ with

$$
\chi_{-}(w)=\frac{1}{2 \pi i} \int_{-i \infty+\varepsilon / 2}^{i \infty+\varepsilon / 2} \frac{\log \psi(z)}{z-w} d z .
$$

See also the results of Rappaport from [31].

The spaces $H^{\infty}(\{s: \operatorname{Re} s<\varepsilon\})$ and $H^{\infty}(\{s:-\varepsilon<\operatorname{Re} s\})$ have intersection equal to the space of bounded entire functions, which is the space $\mathbb{C}$ by Liouville's theorem; hence $\chi_{+}$and $\chi_{-}$are unique up to this additive constant. If $\psi \in G\left(\mathcal{C}_{2}\right)$, then $\psi$ has no zeros and the middle factor is absent, but we are left with the initial factor in (4.4) incorporating the winding number.

On $L^{2}((0, \infty) ; d x)$, let $\mathcal{T}$ be a unitary and self-adjoint operator such that $\mathcal{T}=\mathcal{T}^{\dagger}$ and $\mathcal{T} \mathcal{T}^{\dagger}=I$. Let $Q$ be an orthogonal projection on $L^{2}((0, \infty) ; d x)$, and introduce the complementary spaces $H_{+}=\mathcal{T} Q L^{2}((0, \infty) ; d x)$ and $H_{-}=\mathcal{T}(I-Q) L^{2}((0, \infty) ; d x)$, so $L^{2}=H_{+} \oplus H_{-}$. In the special case of Fourier kernels, and projections onto subintervals, we can identify these subspaces $H_{ \pm}$explicitly. 
Example 4.2. (i) We consider Fourier kernels in the sense of Titchmarsh [38, p. 240]. Let $K \in C([0, \infty)$; $\mathbb{R})$ and suppose that $K_{1}(x)=\int_{0}^{x} K(u) d u$ has $K_{1}(x) / x \in L^{2}(0, \infty)$. Suppose that $K$ has Mellin transform $K^{\star}$ such that $K^{\star}(s) K^{\star}(1-s)=1$ for $\operatorname{Re} s=1 / 2$. Then by Plancherel's theorem, there is an absolutely convergent integral

$$
\int_{0}^{\infty} \frac{K_{1}(x u) K_{1}(u y)}{u^{2}} d u=\min \{x, y\} \quad(x, y>0) .
$$

Then the integral operator $\mathcal{T}: L^{2}(0, \infty) \rightarrow L^{2}(0, \infty)$ given by

$$
\mathcal{T} f(x)=\int_{0}^{\infty} K(x y) f(y) d y
$$

satisfies $\mathcal{T}=\mathcal{T}^{\dagger}$ and $\mathcal{T}^{2}=I$ as in [38, Theorem 133].

(ii) One example of $\mathcal{T}$ is the Fourier cosine transform $C$ where $K(x)=\sqrt{(2 / \pi)} \cos x$. Titchmarsh [38, Theorem 134] gives several other choices, including the Hankel transform $\mathcal{H}_{v} f(x)=\int_{0}^{\infty} \sqrt{x y} J_{v}(x y) f(y) d y$, where $J_{v}$ is Bessel's function of the first kind of order $v \geq-1 / 2$.

Let $L$ be a closed linear subspace of a Hilbert space $H$, and let $T$ be a bounded linear operator on $H$. As in [10, p. 90], we say that $L$ is invariant for $T$ if $T(L) \subseteq L$, and reducing if in addition the orthogonal complement $L^{\perp}=H \ominus L$ satisfies $T\left(L^{\perp}\right) \subseteq L^{\perp}$. It is easy to show that a subspace that is invariant for a unitary group of operators is reducing for the group. This applies to the group $\{I, \mathcal{T}\}$ on $L^{2}(0, \infty)$. Now for $0<a<1<b$, let $Q_{(a, b)}$ be the orthogonal projection $Q_{(a, b)} f(x)=\mathbb{I}_{(a, b)}(x) f(x)$. We write $L^{2}(a, b)=Q_{(a, b)} L^{2}(0, \infty)$ and observe that the partially ordered lattice of subspaces $\left\{L^{2}(a, b): 0<a<1<b<\infty\right\}$ of $L^{2}(0, \infty)$ is unitarily equivalent to the lattice of subspaces

$$
\mathcal{L}=\left\{L^{2}(\alpha, \beta):-\infty<\alpha<0<\beta<\infty\right\}
$$

of $L^{2}(\mathbb{R})$ under the unitary equivalence $f(x) \mapsto e^{-\xi / 2} f\left(e^{-\xi}\right)$. Every subspace in $\mathcal{L}$ is reducing under the unitary group $\left(M_{\lambda}\right)_{\lambda \in \mathbb{R}}$ given by $M_{\lambda} f(x)=e^{i \lambda x} f(x)$, and is also invariant under the unitary dilation semigroup $\left(V_{t}\right)_{t>0}$ where $V_{t} f(x)=e^{-t / 2} f\left(e^{-t} x\right)$. Taken together, these invariance properties characterize $\mathcal{L}$ by [21, p. 104]. The dilations correspond to the scalings $f^{\star}(i \xi+1 / 2) \mapsto e^{t / 2} f^{\star}\left(i e^{t} \xi+1 / 2\right)$ of the Mellin transforms for $f \in L^{2}(0, \infty)$.

Proposition 4.3. Suppose that $K$ is a Fourier kernel as above, and consider $\varphi$ such that

i) $\varphi(z)$ is an entire function of exponential type such that

$$
\begin{aligned}
& \log 1 / a=\lim \sup _{y \rightarrow \infty} \frac{\log |\varphi(i y)|}{y}, \\
& \log 1 / b=-\lim \sup _{y \rightarrow-\infty} \frac{\log |\varphi(i y)|}{|y|} .
\end{aligned}
$$

ii) $\varphi(x) \in L^{2}(\mathbb{R})$.

Then the Mellin transform of the range of $\mathcal{T} Q_{(a, b)}$ is characterized by

$$
\left\{\left(\mathcal{T} Q_{(a, b)} f\right)^{\star}(1 / 2+i z): f \in L^{2}(0, \infty)\right\}=\left\{\varphi(z) K^{\star}(1 / 2+i z): \varphi \quad \text { satisfies }(i),(i i),(4.7)\right\} .
$$

Proof. Now changing variables to $s=1 / 2+i z$, we consider

$$
\begin{aligned}
\varphi(z) & =K^{\star}(1 / 2-i z)\left(\mathcal{T} Q_{(a, b)} f\right)^{\star}(1 / 2+i z) \\
& =\left(Q_{(a, b)} f\right)^{\star}(1 / 2+i z) \\
& =\int_{a}^{b} u^{i z-1 / 2} f(u) d u \\
& =\int_{\log (1 / b)}^{\log (1 / a)} e^{-i x \xi-\xi / 2} f\left(e^{-\xi}\right) d \xi,
\end{aligned}
$$


where the change of variables $u=e^{-\xi}$ gives us a Fourier integral of the function $e^{-\xi / 2} f\left(e^{-\xi}\right)$ in $L^{2}(\log 1 / b, \log 1 / a) \subset L^{2}(\mathbb{R})$, so $\varphi(x) \in L^{2}(\mathbf{R})$. Writing $z=x+i y$, we deduce that $\varphi(z)$ is entire and of enponential type (4.7). By the Paley-Wiener theorem [23, p. 179], these conditions characterize the integrals in (4.8). Thus we characterize the range of $\mathcal{T} Q_{(a, b)}$ via the Mellin transform.

For $g \in L^{\infty}$, let $M_{g} \in \mathcal{L}\left(L^{2}\right)$ be the multiplication operator $M_{g}: h \mapsto g h$. Then we introduce $W_{g} \in \mathcal{L}^{\infty}\left(H_{+}\right)$, $\Gamma_{g} \in \mathcal{L}^{\infty}\left(H_{+}, H_{-}\right),, \tilde{W}_{g} \in \mathcal{L}^{\infty}\left(H_{-}\right), \tilde{\Gamma}_{g} \in \mathcal{L}^{\infty}\left(H_{-}, H_{+}\right)$, by

$$
M_{g}=\left[\begin{array}{cc}
W_{g} & \tilde{\Gamma}_{g} \\
\Gamma_{g} & \tilde{W}_{g}
\end{array}\right] \quad H_{+} .
$$

Lemma 4.4. Let $\mathcal{C}_{p}$ be the space of $g \in L^{\infty}$ such that $\Gamma_{g} \in \mathcal{L}^{p}$ and $\tilde{\Gamma}_{g} \in \mathcal{L}^{p}$, and let

$$
\|g\|_{\mathcal{C}_{p}}=\max \left\{\left\|W_{g}\right\|_{\mathcal{L}^{\infty}},\left\|\tilde{W}_{g}\right\|_{\mathcal{L}^{\infty}}\right\}+\left\|\Gamma_{g}\right\|_{\mathcal{L}^{p}}+\left\|\tilde{\Gamma}_{g}\right\|_{\mathcal{L}^{p}} .
$$

Then $\mathcal{C}_{p}$ is a subalgebra of $L^{\infty}$ such that

$$
\|g h\|_{\mathcal{C}_{p}} \leq\|g\|_{\mathcal{C}_{p}}\|h\|_{\mathcal{C}_{p}}
$$

Proof. For $g \in L^{\infty}$ we have $M_{g} \in \mathcal{L}^{\infty}$, and $\|g\|_{L^{\infty}} \leq\left\|M_{g}\right\|_{\mathcal{L}^{\infty}} \leq\|g\|_{\mathcal{e}_{p}}$, so the pointwise multiplication is unambiguously defined. Conversely, suppose that $g, h \in \mathcal{C}_{p}$, and observe that

$$
\left[\begin{array}{ll}
W_{g h} & \tilde{\Gamma}_{g h} \\
\Gamma_{g h} & \tilde{W}_{g h}
\end{array}\right]=\left[\begin{array}{cc}
W_{g} & \tilde{\Gamma}_{g} \\
\Gamma_{g} & \tilde{W}_{g}
\end{array}\right]\left[\begin{array}{cc}
W_{h} & \tilde{\Gamma}_{h} \\
\Gamma_{h} & \tilde{W}_{h}
\end{array}\right]=\left[\begin{array}{ll}
W_{g} W_{h}+\tilde{\Gamma}_{g} \Gamma_{h} & W_{g} \tilde{\Gamma}_{h}+\tilde{\Gamma}_{g} \tilde{W}_{h} \\
\Gamma_{g} W_{h}+\tilde{W}_{g} \Gamma_{h} & \tilde{W}_{g} \tilde{W}_{h}+\Gamma_{g} \tilde{\Gamma}_{h}
\end{array}\right]
$$

leading to identities such as

$$
\begin{gathered}
W_{g h}=W_{g} W_{h}+\tilde{\Gamma}_{g} \Gamma_{h} \\
\Gamma_{g h}=\Gamma_{g} W_{h}+\tilde{W}_{g} \Gamma_{h} .
\end{gathered}
$$

The ideal property of the Schatten norm gives

$$
\begin{gathered}
\left\|W_{g h}\right\|_{\mathcal{L}^{\infty}} \leq\left\|W_{g}\right\|_{\mathcal{L}^{\infty}}\left\|W_{h}\right\|_{\mathcal{L}^{\infty}}+\left\|\tilde{\Gamma}_{g}\right\|_{\mathcal{L}^{p}}\left\|\Gamma_{h}\right\|_{\mathcal{L}^{p}}, \\
\left\|\Gamma_{g h}\right\|_{\mathcal{L}^{\infty}} \leq\left\|\Gamma_{g}\right\|_{\mathcal{L}^{p}}\left\|W_{h}\right\|_{\mathcal{L}^{\infty}}+\left\|\tilde{W}_{g}\right\|_{\mathcal{L}^{\infty}}\left\|\Gamma_{h}\right\|_{\mathcal{L}^{p}},
\end{gathered}
$$

and similar inequalities for each entry of (4.12), hence the norm satisfies the submultiplicative property.

Let $\mathcal{A}_{2}$ be the subalgebra of $\mathcal{C}_{2}$ consisting of $f \in \mathcal{C}_{2}$ such that $f$ is bounded and holomorphic on the right half plane, and let $\mathcal{A}_{2}^{\star}$ be the subalgebra of $\mathcal{C}_{2}$ consisting of $f \in \mathcal{C}_{2}$ such that $f(z)=\bar{g}(-\bar{z})$ for some $g \in \mathcal{A}_{2}$. Here $f^{\star}$ denotes the reflection of $f$, in the sense of Schwarz's reflection principle. Note that $\mathcal{A}_{2}^{*} \cap \mathcal{A}_{2}=\mathbb{C} 1$ by Liouville's theorem. The following result describes $\psi \in G\left(\mathcal{A}_{2}^{*}\right) G\left(\mathcal{A}_{2}\right)$ that has no imaginary zeros, but may have zeros elsewhere. For $\mathcal{G}$ a group, we write $\{X, Y\}=X Y X^{-1} Y^{-1}$ for the multiplicative commutator of $X, Y \in \mathcal{G}$.

Lemma 4.5. Suppose that $\psi \in \mathcal{C}_{2}$ has no zeros on the imaginary axis,

(1) $\psi(i \xi+\eta) \rightarrow 1$ as $\xi \rightarrow \pm \infty$, uniformly for $-\varepsilon<\eta<\varepsilon$,

(2) the winding number of $\{\psi(i \xi):-\infty \leq \xi \leq \infty\}$ is zero, and

(3) $\psi(z)=1+O\left(1 /|z|^{1 / 2+\delta}\right)$ as $|z| \rightarrow \infty$ for some $0<\delta \leq 1 / 2$.

Then there exists $0<\varepsilon^{\prime}<\varepsilon$ such that $\psi$ has a Wiener-Hopf factorization

$$
\psi=\psi_{-} \psi_{+} \quad\left(-\varepsilon^{\prime}<\operatorname{Re} z<\varepsilon^{\prime}\right)
$$

such that 
(i) $\psi$ - is bounded, holomorphic and free from zeros on $\left\{z: \operatorname{Re} z<\varepsilon^{\prime} / 2\right\}$;

(ii) $\psi_{+}$is bounded, holomorphic and free from zeros on $\left\{z: \operatorname{Re} z>-\varepsilon^{\prime} / 2\right\}$;

(iii) $\psi_{ \pm}(\eta+i \xi)=1+O\left(1 /|z|^{(1+\delta) / 2}\right)$ as $\xi \rightarrow \pm \infty$, uniformly for $-\varepsilon^{\prime} / \varepsilon<\eta<\varepsilon^{\prime} / \varepsilon$.

Proof. (i), (ii) By hypothesis, $\psi$ has no zeros lie on the imaginary axis, and only finitely many in the strip $\{z:-\varepsilon<\operatorname{Re} z<\varepsilon\}$; so by choosing $0<\varepsilon^{\prime}<\varepsilon$ sufficiently small, we can ensure that $\psi$ is free from zeros $\{z:-\varepsilon \leq \operatorname{Re} z \leq \varepsilon\}$. Then we choose

$$
\begin{gathered}
\chi_{-}(z)=\int_{-i \infty+\varepsilon^{\prime}}^{i \infty+\varepsilon} \frac{\log \psi(z)}{z-w} d z, \\
\chi_{+}(z)=\frac{1}{2 \pi i} \int_{-i \infty-\varepsilon^{\prime}}^{i \infty-\varepsilon^{\prime}} \frac{\log \psi(z)}{z-w} d z ;
\end{gathered}
$$

then the functions $\psi_{-}(z)=\exp \left(-\chi_{-}(z)\right)$ and $\psi_{+}(z)=\exp \left(\chi_{+}(z)\right)$ satisfy $\psi_{-} \psi_{+}=\psi$, as in (4.17). Also, we can introduce $R>0$ such that

$$
\sup \left\{|\psi(\eta+i \xi)-1|:-\varepsilon^{\prime}<\eta<\varepsilon^{\prime}, \xi \in(-\infty,-R) \cup(R, \infty)\right\}<1 / 2
$$

and $\psi(z)$ is free from zeros on $\left\{z=\eta+i \xi:-\varepsilon^{\prime}<\eta<\varepsilon^{\prime}, \xi \in[-R, R]\right\}$. Then one can introduce $M$ such that

$$
|\log \psi(z)| \leq \frac{M}{(1+|\xi|)^{1 / 2+\delta}} \quad\left(z=\eta+i \xi ;-\infty<\xi<\infty, \quad-\varepsilon^{\prime}<\eta<\varepsilon^{\prime}\right) .
$$

The convolution of a pair of $L^{2}$ functions gives a continuous function which vanishes at infinity, so $\chi_{ \pm}$are bounded and holomorphic on the smaller half planes determined by abscissae $\pm \varepsilon^{\prime} / 2$.

(iii) To obtain the more precise estimate of (iii), we consider $z$ with $-\varepsilon^{\prime} / 2 \leq \operatorname{Re} z \leq \varepsilon^{\prime} / 2$ and $\operatorname{Im} z>0$ large; then we take $\xi_{0}=|z| / 2$ and $p>2 / \delta$ with conjugate $q=p /(p-1)$ and split the integral

$$
\begin{aligned}
\left|\chi_{-}(z)\right| & \leq \int_{-\infty}^{\infty} \frac{\left|\log \psi\left(\varepsilon^{\prime}+i \xi\right)\right|}{\left|z-\varepsilon^{\prime}-i \xi\right|} d \xi \\
& =\int_{-\infty}^{\xi_{0}}+\int_{\xi_{0}}^{\infty} \frac{\left|\log \psi\left(\varepsilon^{\prime}+i \xi\right)\right|}{\left|z-\varepsilon^{\prime}-i \xi\right|} d \xi \\
& \leq\left(\int_{-\infty}^{\xi_{0}} \frac{M^{q}}{(1+|\xi|)^{q / 2+q \delta}} d \xi\right)^{1 / q}\left(\int_{-\infty}^{\xi_{0}} \frac{d \xi}{\left|z-\varepsilon^{\prime}-i \xi\right|^{p}}\right)^{1 / p}+\left(\int_{\xi_{0}}^{\infty} \frac{M^{p}}{(1+|\xi|)^{p / 2+p \delta}} d \xi\right)^{1 / p}\left(\int_{\xi_{0}}^{\infty} \frac{d \xi}{\left|z-\varepsilon^{\prime}-i \xi\right|^{q}}\right)^{1 / q} \\
& =O\left(1 /|z|^{1-1 / p}\right)+O\left(1 /|z|^{1 / 2+\delta-1 / p}\right),
\end{aligned}
$$

where we have used Hölder's inequality on the integrals. The other estimates in (iii) are similar. Likewise, one can show that $\chi_{ \pm}^{\prime}(z)=O\left(1 /|z|^{(1+\delta) / 2}\right)$ as $\left.\xi \rightarrow \pm \infty\right)$.

\section{Wiener-Hopf determinant}

This section contains the main theoretical result, as follows. 
Theorem 5.1. Suppose that $\psi \in L^{\infty}(i \mathbb{R})$ has a Wiener-Hopf factorization $\psi=\psi_{-} \psi_{+}$as in Lemma 4.5. Then there exists a $2 \times 2$ scattering function

$$
\Phi(x)=\left[\begin{array}{cc}
0 & \phi_{1}(x) \\
\phi_{2}(x) & 0
\end{array}\right]
$$

such that Hankel operators integral operators $\Gamma_{\phi_{1}}$ and $\Gamma_{\phi_{2}}$ are Hilbert-Schmidt on $L^{2}(0, \infty)$ and

$$
1 / \operatorname{det}\left(W(\psi) W\left(\psi^{-1}\right)\right)=\operatorname{det}\left(I-\Gamma_{\phi_{1}} \Gamma_{\phi_{2}}\right)=\operatorname{det}_{2}\left(I+\Gamma_{\Phi}\right) \text {. }
$$

There are three particular cases that arise under the following hypotheses:

(i) $\psi_{-}(i \xi) / \bar{\psi}_{-}(-i \xi)=\psi_{+}(i \xi) / \bar{\psi}_{+}(-i \xi)$ if and only if $\phi_{1}$ and $\phi_{2}$ are real, so that $\Gamma_{\phi_{1}}$ and $\Gamma_{\phi_{2}}$ are self-adjoint;

(ii) $\psi_{-}(i \xi) \psi_{-}(-i \xi)=\psi_{+}(i \xi) \psi_{+}(-i \xi)$, if and only if $\phi_{1}=\phi_{2}$, in which case the Carleman determinants satisfy

$$
\operatorname{det}_{2}\left(I+\lambda \Gamma_{\Phi}\right)=\operatorname{det}_{2}\left(I-\lambda \Gamma_{\phi_{1}}\right) \operatorname{det}_{2}\left(I+\lambda \Gamma_{\phi_{1}}\right) \quad(\lambda \in \mathbb{C})
$$

(iii) $\left|\psi_{-}(i \xi)\right|=\left|\psi_{+}(i \xi)\right|$ if and only if the operator $\Gamma_{\Phi}$ is self-adjoint.

Any pair of these conditions implies the other one.

Proof. By the Lemma 4.5, we can choose $\varepsilon^{\prime}>0$ such that

$$
f=\frac{\psi_{-}}{\psi_{+}}-1, \quad g=\frac{\psi_{+}}{\psi_{-}}-1
$$

both belong to $\mathcal{C}_{2}=\mathcal{C}_{2}\left(\varepsilon^{\prime}\right)$ and satisfy $f g=2-\psi_{-} / \psi_{+}-\psi_{+} / \psi_{-}$; hence

$$
\begin{aligned}
\tilde{\Gamma}(f) \Gamma(g) & =W(f g)-W(f) W(g) \\
& =I-W\left(\psi_{-} / \psi_{+}\right) W\left(\psi_{+} / \psi_{-}\right) .
\end{aligned}
$$

Now the operators $W\left(\psi_{ \pm}\right)$are invertible, and $W\left(\rho \psi_{+}\right)=W(\rho) W\left(\psi_{+}\right)$and $W\left(\psi_{-} \rho\right)=W\left(\psi_{-}\right) W(\rho)$ for all $\rho \in \mathcal{C}_{2}$. So using some identities from [2], we have

$$
\begin{aligned}
W\left(\psi_{-} / \psi_{+}\right) W\left(\psi_{+} / \psi_{-}\right) & =W\left(\psi_{-}\right) W\left(\psi_{+}\right)^{-1} W\left(\psi_{+} / \psi_{-}\right) \\
& =W\left(\psi_{-}\right) W\left(\psi_{+}\right)^{-1} W\left(\psi_{-}\right)^{-1} W\left(\psi_{+}\right) \\
& =\left\{W\left(\psi_{-}\right), W\left(\psi_{+}\right)^{-1}\right\}
\end{aligned}
$$

so taking the determinant of the inverse of the right-hand side, we have

$$
\operatorname{det}\left\{W\left(\psi_{-}\right), W\left(\psi_{+}\right)^{-1}\right\}=\operatorname{det}(I-\tilde{\Gamma}(f) \Gamma(g)) \text {. }
$$

We also have

$$
W\left(\psi_{-}\right) W\left(\psi_{+}\right)^{-1} W\left(\psi_{-}\right)^{-1} W\left(\psi_{+}\right)=W\left(\psi_{-}\right) W\left(\psi_{-} \psi_{+}\right)^{-1} W\left(\psi_{+}\right)
$$

so

$$
\begin{aligned}
\operatorname{det}\left(W\left(\psi_{-}\right) W\left(\psi_{+}\right)^{-1} W\left(\psi_{-}\right)^{-1} W\left(\psi_{+}\right)\right) & =1 / \operatorname{det}\left(W\left(\psi_{+}\right)^{-1} W\left(\psi_{-} \psi_{+}\right) W\left(\psi_{-}\right)^{-1}\right) \\
& =1 / \operatorname{det}\left(W\left(\psi_{-}\right)^{-1} W\left(\psi_{+}\right)^{-1} W\left(\psi_{-} \psi_{+}\right)\right) \\
& =1 / \operatorname{det}\left(W\left(\psi_{-}^{-1} \psi_{+}^{-1}\right) W\left(\psi_{-} \psi_{+}\right)\right) .
\end{aligned}
$$

Taking the unitary conjugation by the Fourier transform, we have $\tilde{\Gamma}(f) \mapsto \Gamma_{\phi_{1}}$ and $\Gamma(g) \mapsto \Gamma_{\phi_{2}}$, where

$$
\phi_{1}(x)=\int_{-\infty}^{\infty}\left(\frac{\psi_{-}(i \xi)}{\psi_{+}(i \xi)}-1\right) e^{-i \xi x} d \xi
$$




$$
\phi_{2}(x)=\int_{-\infty}^{\infty}\left(\frac{\psi_{+}(-i \xi)}{\psi_{-}(-i \xi)}-1\right) e^{-i \xi x} d \xi
$$

the difference in signs $\pm \xi$ in the quotients reflecting the tilde on $\tilde{\Gamma}(f)$.

Hence $\psi_{-} / \psi_{+}-1$ and $\psi_{+} / \psi_{-}-1$ belong to $L^{2}(i \mathbb{R}) \cap L^{\infty}(i \mathbb{R})$ and determine bounded Hankel operators. We proceed to realize these via linear systems. Let $H=L^{2}(-\infty, \infty)$ and $\mathcal{D}(A)=\{g \in H: \xi g(\xi) \in H\}$. Then we introduce the linear systems $\left(-A, B_{1}, C\right)$ and $\left(-A, B_{2}, C\right)$ by

$$
\begin{array}{cl}
A: \mathcal{D}(A) \rightarrow H: & g(\xi) \mapsto i \xi g(\xi) \quad(g \in \mathcal{D}(A)) ; \\
B_{1}: \mathbb{C} \rightarrow H: & \beta \mapsto\left(\frac{\psi_{-}(i \xi)}{\psi_{+}(i \xi)}-1\right) \beta \quad(\beta \in \mathbb{C}) ; \\
B_{2}: \mathbb{C} \rightarrow H: & \beta \mapsto\left(\frac{\psi_{+}(-i \xi)}{\psi_{-}(-i \xi)}-1\right) \beta \quad(\beta \in \mathbb{C}) ; \\
C: \mathcal{D}(A) \rightarrow \mathbb{C}: & g \mapsto \frac{1}{2 \pi} \int_{-\infty}^{\infty} g(\xi) d \xi \quad(g \in \mathcal{D}(A)) .
\end{array}
$$

Then $-A$ generates the unitary group $\left(e^{-t A}\right)_{t \in \mathbb{R}}$ where $e^{-t A}: g(\xi) \mapsto e^{-i t \xi} g(\xi)$ and by (5.10) and (5.11), we have $\phi_{1}(t)=C e^{-t A} B_{1}$ and $\phi_{2}(t)=C e^{-t A} B_{2}$. Also, $\Gamma_{\phi_{1}}$ and $\Gamma_{\phi_{2}}$ are Hilbert-Schmidt by Proposition 2.2. Hence $\Gamma_{\phi_{1}} \Gamma_{\phi_{2}}$ is a trace class operator, and $\operatorname{det}\left(I-\Gamma_{\phi_{1}} \Gamma_{\phi_{2}}\right)$ is well defined.

Suppose that the linear system $\left(-A, B_{j}, C\right)$ realizes $\phi_{j}$. Then the matrix system

$$
\left(\left[\begin{array}{cc}
-A & 0 \\
0 & -A
\end{array}\right],\left[\begin{array}{cc}
B_{1} & 0 \\
0 & B_{2}
\end{array}\right],\left[\begin{array}{ll}
0 & C \\
C & 0
\end{array}\right]\right)
$$

realizes

$$
\Phi(x)=\left[\begin{array}{cc}
0 & \phi_{1}(x) \\
\phi_{2}(x) & 0
\end{array}\right]
$$

For finite matrices $U$ and $V$, we have

$$
\operatorname{det}\left[\begin{array}{cc}
I & 0 \\
0 & I-U V
\end{array}\right]=\operatorname{det}_{2}\left[\begin{array}{cc}
I & 0 \\
-V & I
\end{array}\right] \operatorname{det}\left[\begin{array}{cc}
I & U \\
V & I
\end{array}\right] \operatorname{det}_{2}\left[\begin{array}{cc}
I & -U \\
0 & I
\end{array}\right]
$$

so by a simple approximation argument in Hilbert-Schmidt norm

$$
\operatorname{det}\left(I-\Gamma_{\phi_{1}} \Gamma_{\phi_{2}}\right)=\operatorname{det}\left(I+\Gamma_{\Phi}\right)
$$

Hence

$$
1 / \operatorname{det}\left(W(\psi) W\left(\psi^{-1}\right)\right)=\operatorname{det}_{2}\left(I+\Gamma_{\Phi}\right) .
$$

(i) Now by uniqueness of the Fourier transform, $\phi_{1}$ is real if and only if $\psi_{-} / \psi_{+}(i \xi)=\bar{\psi}_{-}(-i \xi) / \bar{\psi}_{+}(-i \xi)$.

(ii) Likewise $\phi_{1}(x)=\phi_{2}(x)$ if and only if $\psi_{-}(i \xi) / \psi_{+}(i \xi)=\psi_{+}(-i \xi) / \psi_{-}(-i \xi)$, which reduces to the stated condition. If $\phi_{1}=\phi_{2}$, then

$$
\operatorname{det}\left(I+\lambda \Gamma_{\Phi}\right)=\operatorname{det}\left(I-\lambda^{2} \Gamma_{\phi_{1}}^{2}\right)=\operatorname{det}\left(I-\lambda \Gamma_{\phi_{1}}\right) \operatorname{det}\left(I+\lambda \Gamma_{\phi_{1}}\right)
$$

is determined by the spectrum of the scalar-valued Hankel operator $\Gamma_{\phi_{1}}$. The nature of the spectrum is determined in [25] and [29].

(iii) Evidently $\Gamma_{\Phi}$ is self-adjoint if and only if $\bar{\phi}_{1}(x)=\phi_{2}(x)$; that is

$$
\bar{\psi}_{-}(-i \xi) / \bar{\psi}_{+}(-i \xi)=\psi_{+}(-i \xi) / \psi_{-}(-i \xi) \text {. }
$$

Finally, one considers the cases (i), (ii) and (iii) as they apply to $\left[\begin{array}{ll}0 & U \\ V & 0\end{array}\right]$. 
As in Corollary 3.3, we can reduce the Fredholm determinant of Hankel operators to related determinants. Let $P$ and $Q$ be orthogonal projections on $L^{2}(0, \infty)$ such that $P+Q=I$. Then

$$
\begin{aligned}
\operatorname{det}\left(P\left\{W\left(\psi_{+}\right)^{-1}, W\left(\psi_{-}\right)\right\} P\right) & =\operatorname{det}\left\{W\left(\psi_{+}\right)^{-1}, W\left(\psi_{-}\right)\right\} \operatorname{det}\left(Q\left\{W\left(\psi_{-}\right), W\left(\psi_{+}\right)^{-1}\right\} Q\right) \\
& =\operatorname{det}(I-\tilde{\Gamma}(f) \Gamma(g))^{-1} \operatorname{det}(Q-Q \tilde{\Gamma}(f) \Gamma(g) Q) .
\end{aligned}
$$

Self-adjoint block Hankel matrices have been characterized up to unitary equivalence, as in [25, Theorem 2].

Corollary 5.2. Let $a_{j}, b_{j}, c_{j}, d_{j} \in(0, \infty)$ and

$$
\psi(i \xi)=\prod_{j=1}^{m} \frac{\Gamma\left(a_{j}+i \xi\right)}{\Gamma\left(b_{j}+i \xi\right)} \prod_{j=1}^{\mu} \frac{\Gamma\left(c_{j}-i \xi\right)}{\Gamma\left(d_{j}-i \xi\right)}
$$

where the zeros and poles satisfy

$$
\sum_{j=1}^{m}\left(a_{j}-b_{j}\right)=0=\sum_{j=1}^{\mu}\left(c_{j}-d_{j}\right)
$$

(i) Then there exists a linear system as in (5.12) such that

$$
1 / \operatorname{det}\left(W(\psi) W\left(\psi^{-1}\right)\right)=\operatorname{det}_{2}\left(I+\Gamma_{\Phi}\right) .
$$

Also, $\phi_{1}$ and $\phi_{2}$ are real.

(ii) Suppose further that $m=\mu$ and $a_{j}=c_{j}$ and $b_{j}=d_{j}$ for $j=1, \ldots, m$. Then $\phi_{1}=\phi_{2}$ and $\Gamma_{\Phi}$ is self-adjoint.

Proof. (i) The following analysis is suggested by the discussion of Barnes-Mellin integrals from [12, p. 49], although it differs in detail. For $j=1, \ldots, m$, let $a_{j}, b_{j} \in(0, \infty)$ be such that $\sum_{j=1}^{m}\left(a_{j}-b_{j}\right)=0$. Then

$$
\psi_{-}(i \xi)=\prod_{j=1}^{m} \frac{\Gamma\left(a_{j}+i \xi\right)}{\Gamma\left(b_{j}+i \xi\right)}
$$

is meromorphic with poles at $\xi=i a_{j}, i a_{j}+i, i a_{j}+2 i, \ldots$ and zeros at $\xi=i b_{j}, i b_{j}+i, i b_{j}+2 i, \ldots$, all in the open upper half plane. For $0<\varepsilon<\pi$, Stirling's formula from [37, p. 151] gives

$$
\log \Gamma(z)=(z-1 / 2) \log z-z+\log \sqrt{2 \pi}+\frac{1}{12 z}+O\left(1 /|z|^{2}\right)
$$

as $z \rightarrow \infty$ with $-\pi+\varepsilon<\arg z<\pi-\varepsilon$, hence

$$
\begin{aligned}
\log \psi_{-}(i \xi)= & \sum_{j=1}^{m}\left(\left(i \xi+a_{j}-1 / 2\right) \log \left(i \xi+a_{j}\right)-\left(i \xi+b_{j}-1 / 2\right) \log \left(i \xi+b_{j}\right)\right) \\
& -\sum_{j=1}^{m}\left(\left(i \xi+a_{j}-1 / 2\right)-\left(i \xi+b_{j}-1 / 2\right)\right)+\frac{1}{12} \sum_{j=1}^{m}\left(\frac{1}{i \xi+a_{j}}-\frac{1}{i \xi+b_{j}}\right)+O\left(1 / \xi^{2}\right)
\end{aligned}
$$

where the second sum vanishes, and the third one is small, so we have

$$
\log \psi_{-}(i \xi)=\sum_{j=1}^{m}\left[\left(i \xi+a_{j}-1 / 2\right) \log \left(1-\frac{i a_{j}}{\xi}\right)-\left(i \xi+b_{j}-1 / 2\right) \log \left(1-\frac{i b_{j}}{\xi}\right)\right]+O\left(1 / \xi^{2}\right)
$$

which by the Maclaurin series for the logarithms gives

$$
=\sum_{j=1}^{m}\left[\left(i \xi+a_{j}-1 / 2\right)\left(\frac{-i a_{j}}{\xi}+\frac{a_{j}^{2}}{2 \xi^{2}}+O\left(1 / \xi^{3}\right)\right)-\left(i \xi+b_{j}-1 / 2\right)\left(\frac{-i b_{j}}{\xi}+\frac{b_{j}^{2}}{2 \xi^{2}}+O\left(1 / \xi^{3}\right)\right)\right]+O\left(1 / \xi^{2}\right)
$$

hence we obtain the asymptotic formula

$$
\log \psi_{-}(i \xi)=\log \left|\psi_{-}(i \xi)\right|+i \arg \psi_{-}(i \xi)=-\frac{i}{2 \xi} \sum_{j=1}^{m}\left(a_{j}^{2}-b_{j}^{2}\right)+O\left(1 / \xi^{2}\right) \quad(\xi \rightarrow \pm \infty),
$$


so

$$
\psi_{-}(i \xi)=1-\frac{i}{2 \xi} \sum_{j=1}^{m}\left(a_{j}^{2}-b_{j}^{2}\right)+O\left(1 / \xi^{2}\right) \quad(\xi \rightarrow \pm \infty)
$$

as $\xi \rightarrow \pm \infty$ along the real axis. Likewise, for $j=1, \ldots, \mu$, let $c_{j}, d_{j} \in(0, \infty)$ be non zero real numbers such that $\sum_{j=1}^{\mu}\left(c_{j}-d_{j}\right)=0$. Then

$$
\psi_{+}(i \xi)=\prod_{j=1}^{\mu} \frac{\Gamma\left(c_{j}-i \xi\right)}{\Gamma\left(d_{j}-i \xi\right)}
$$

is meromorphic with poles at $-i c_{j},-i c_{j}-i,-i c_{j}-2 i, \ldots$ and zeros at $-i d_{j},-i d_{j}-i,-i d_{j}-2 i, \ldots$, all in open lower half plane, and

$$
\psi_{+}(i \xi)=1+\frac{i}{2 \xi} \sum_{j=1}^{\mu}\left(c_{j}^{2}-d_{j}^{2}\right)+O\left(1 / \xi^{2}\right) \quad(\xi \rightarrow \pm \infty)
$$

as $\xi \rightarrow \pm \infty$ along the real axis.

Hence $\psi_{-} / \psi_{+}-1$ and $\psi_{+} / \psi_{-}-1$ belong to $L^{2}(i \mathbb{R})$ and $L^{\infty}(i \mathbb{R})$ and determine Hankel operators. We proceed to realize these via linear systems. Let $H=L^{2}(-\infty, \infty)$ and $\mathcal{D}(A)=\{g \in H: \xi g(\xi) \in H\}$. Then we introduce the linear systems $\left(-A, B_{1}, C\right)$ and $\left(-A, B_{2}, C\right)$ by

$$
\begin{aligned}
A: \mathcal{D}(A) \rightarrow H: & g(\xi) \mapsto i \xi g(\xi) \quad(g \in \mathcal{D}(A)) ; \\
B_{1}: \mathbb{C} \rightarrow H: & \beta \mapsto\left(\frac{\psi_{-}(i \xi)}{\psi_{+}(i \xi)}-1\right) \beta \quad(\beta \in \mathbb{C}) ; \\
B_{2}: C \rightarrow H: & \beta \mapsto\left(\frac{\psi_{+}(-i \xi)}{\psi_{-}(-i \xi)}-1\right) \beta \quad(\beta \in \mathbb{C}) ; \\
C: \mathcal{D}(A) \rightarrow \mathbb{C}: & g \mapsto \frac{1}{2 \pi} \int_{-\infty}^{\infty} g(\xi) d \xi \quad(g \in \mathcal{D}(A)) .
\end{aligned}
$$

Then $\phi_{1}(t)=C e^{-t A} B_{1}$ and $\phi_{2}(t)=C e^{-t A} B_{2}$. From the formula

$$
i x \phi_{1}(x)=\frac{1}{2 \pi} \int_{-\infty}^{\infty} e^{-i x \xi} \frac{\psi_{-}(i \xi)}{\psi_{+}(i \xi)} \frac{d}{d \xi}\left(\log \psi_{-}(i \xi)-\log \psi_{+}(i \xi)\right) d \xi
$$

we deduce that $\phi_{1}(x)$ and $x \phi_{1}(x)$ belong to $L^{2}(0, \infty)$, hence $\Gamma_{\phi_{1}}$ is Hilbert-Schmidt; likewise $\Gamma_{\phi_{2}}$ is HilbertSchmidt. Hence $\Gamma_{\phi_{1}} \Gamma_{\phi_{2}}$ is a trace class operator, and $\operatorname{det}\left(I-\Gamma_{\phi_{1}} \Gamma_{\phi_{2}}\right)$ is well defined.

Here we have $\psi_{-}(i \xi)=\bar{\psi}_{-}(-i \xi)$ and $\psi_{+}(i \xi)=\bar{\psi}_{+}(-i \xi)$, so $\phi_{1}$ and $\phi_{2}$ are real.

(ii) This is a special case of (ii) of the Theorem 5.1.

\section{Determinant expansions}

In case (ii) of the Corollary 5.2 we can compute $\phi_{1}$ and $\phi_{2}$ explicitly. The theorem [29, Theorem 1.4 page 237] shows that a Hankel operator is trace class if and only if it has a nuclear expansion as a series of Hankel operators of rank one. So to compute $\Gamma_{\phi_{1}}$ and $\Gamma_{\phi_{2}}$ as trace class operators on $L^{2}(x, \infty)$, we select a sequence of exponential functions $\left(e^{-\lambda_{j} t}\right)_{j=0}^{\infty}$ in $L^{2}(x, \infty)$ so that $\Gamma_{\phi_{1}}$ has a nuclear expansion in terms of rank one Hankel operators; ultimately, this will enable us to compute the determinant of $I-\Gamma_{\phi_{1}} \Gamma_{\phi_{2}}$ compressed to $L^{2}(x, \infty)$ in terms of an infinite matrix. For large $x$, most of the entries of this matrix are very small, so this is a practicable means for computing the determinant. Our method follows [7].

In the following calculation, we use the generalized hypergeometric function $2 m F_{2 m-1}[; z]$ which has a convergent power series expansion for all $|z|<1$ by [12, p. 182]. For comparison, [2] uses ${ }_{4} F_{3}$. Let $s=i \xi$ and 
$z=e^{-x}$ where $\operatorname{Re} x>0$ so $|z|<1$. We consider

$$
\begin{aligned}
\phi_{1}(x) & =\frac{1}{2 \pi} \int_{-\infty}^{\infty}\left(\frac{\psi_{-}(i \xi)}{\psi_{+}(i \xi)}-1\right) e^{-i \xi x} d \xi \\
& =\frac{1}{2 \pi i} \int_{-i \infty}^{i \infty}\left(\prod_{\ell=1}^{m} \frac{\Gamma\left(a_{\ell}+s\right) \Gamma\left(d_{\ell}-s\right)}{\Gamma\left(b_{\ell}+s\right) \Gamma\left(c_{\ell}-s\right)}-1\right) z^{-s} d s \\
& =\frac{1}{2 \pi i} \int_{-i \infty}^{i \infty}\left(\prod_{\ell=1}^{m} \frac{\Gamma\left(1-b_{\ell}-s\right) \operatorname{cosec} \pi\left(a_{\ell}+s\right) \Gamma\left(d_{\ell}-s\right)}{\Gamma\left(1-a_{\ell}-s\right) \operatorname{cosec} \pi\left(b_{\ell}+s\right) \Gamma\left(c_{\ell}-s\right)}-1\right) z^{-s} d s,
\end{aligned}
$$

where we have used the formula $\Gamma(w) \Gamma(1-w)=\pi \operatorname{cosec} \pi w$; now we take an integral round a semicircular contour in the left half plane and sum over the residues at poles near the negative real axis of $s$ to obtain

$$
\begin{aligned}
\phi_{1}(x) & =\sum_{j=1}^{m} \sum_{k=0}^{\infty} \operatorname{Res}\left(-a_{j}-k\right) \\
& =\sum_{j=1}^{m} \sum_{k=0}^{\infty} \frac{(-1)^{k}}{\pi \Gamma(1+k)} \prod_{\ell=1 ; \ell \neq j}^{m} \frac{\operatorname{cosec} \pi\left(a_{\ell}-a_{j}-k\right)}{\Gamma\left(1-a_{\ell}+a_{j}+k\right)} \prod_{\ell=1}^{m} \frac{\Gamma\left(1-b_{\ell}+a_{j}+k\right) \Gamma\left(d_{\ell}+a_{j}+k\right)}{\operatorname{cosec} \pi\left(b_{\ell}-a_{j}-k\right) \Gamma\left(c_{\ell}+a_{j}+k\right)} z^{a_{j}+k},
\end{aligned}
$$

where we have picked out the factor $\operatorname{cosec} \pi\left(s_{j}+s\right) / \Gamma\left(1-a_{j}-s\right)$ that contributes the pole, so

$$
\begin{aligned}
& \phi_{1}(x) \\
& =\sum_{j=1}^{m} \frac{1}{\pi} \prod_{\ell: \ell \neq j} \frac{\operatorname{cosec} \pi\left(a_{\ell}-a_{j}\right)}{\Gamma\left(1-a_{\ell}+a_{j}\right)} \prod_{\ell=1}^{m} \frac{\Gamma\left(1-b_{\ell}+a_{j}\right) \Gamma\left(d_{\ell}+a_{j}\right)}{\operatorname{cosec} \pi\left(b_{\ell}-a_{j}\right) \Gamma\left(c_{\ell}+a_{j}\right)} \sum_{k=0}^{\infty} \prod_{\ell=1}^{m} \frac{\left(1-b_{\ell}+a_{j}\right)_{k}\left(d_{\ell}+a_{j}\right)_{k}}{\left(1-a_{\ell}+a_{j}\right)_{k}\left(c_{\ell}+a_{j}\right)_{k}} z^{a_{j}+k}, \\
& =\sum_{j=1}^{m} \frac{1}{\pi} \prod_{\ell: \ell \neq j} \frac{\operatorname{cosec} \pi\left(a_{\ell}-a_{j}\right)}{\Gamma\left(1-a_{\ell}+a_{j}\right)} \prod_{\ell=1}^{m} \frac{\Gamma\left(1-b_{\ell}+a_{j}\right) \Gamma\left(d_{\ell}+a_{j}\right)}{\operatorname{cosec} \pi\left(b_{\ell}-a_{j}\right) \Gamma\left(c_{\ell}+a_{j}\right)} z^{a_{j}} \\
& \times{ }_{2 m} F_{2 m-1}\left[\begin{array}{crrr}
1-b_{1}+a_{j} \ldots 1-b_{j}+a_{j} \ldots 1-b_{m}+a_{j} & d_{1}+a_{j} \ldots d_{j}+a_{j} \ldots d_{m}+a_{j} \\
1-a_{1}+a_{j} \ldots & \hat{1} & \ldots 1-a_{m}+a_{j} & c_{1}+a_{j} \ldots c_{j}+d_{j} \ldots c_{m}+a_{j}
\end{array} ;,,\right.
\end{aligned}
$$

where, with Pochhammer's rising factorial,

$$
z=e^{-x}, \quad(c)_{0}=1, \quad(c)_{k}=c(c+1) \ldots(c+k-1),
$$

and $\hat{1}$ stands for the omitted term in the denominator, and we have written this expression in terms of the generalized hypergeometric functions ${ }_{2 m} F_{2 m-1}[; z]$, as in [12, page 182]. There is a similar formula for $\phi_{2}(z)$ in which $\left(c_{j}, d_{j}, b_{j}, a_{j}\right)$ replaces $\left(a_{j}, b_{j}, d_{j}, c_{j}\right)$.

Without loss of generality, we suppose $a_{1}<a_{2}<\cdots<a_{m}$, so taking the term from $\operatorname{Res}\left(-a_{1}\right)$ and using the convergent series (6.4), we have

$$
\phi_{1}(x)=\frac{1}{\pi} \prod_{\ell=2}^{m} \frac{\operatorname{cosec} \pi\left(a_{\ell}-a_{1}\right)}{\Gamma\left(1-a_{\ell}+a_{1}\right)} \prod_{\ell=1}^{m} \frac{\Gamma\left(1-b_{\ell}+a_{1}\right) \Gamma\left(d_{\ell}+a_{1}\right)}{\operatorname{cosec} \pi\left(b_{\ell}-a_{1}\right) \Gamma\left(c_{\ell}+a_{1}\right)} e^{-a_{1} x}+O\left(e^{-a_{2} x}+e^{-\left(a_{1}+1\right) x}\right),
$$

and likewise with $c_{1}<c_{2}<\cdots<c_{m}$, taking the term from $\operatorname{Res}\left(-c_{1}\right)$, we have

$$
\phi_{2}(x)=\frac{1}{\pi} \prod_{\ell=2}^{m} \frac{\operatorname{cosec} \pi\left(c_{\ell}-c_{1}\right)}{\Gamma\left(1-c_{\ell}+c_{1}\right)} \prod_{\ell=1}^{m} \frac{\Gamma\left(1-d_{\ell}+c_{1}\right) \Gamma\left(b_{\ell}+c_{1}\right)}{\operatorname{cosec} \pi\left(d_{\ell}-c_{1}\right) \Gamma\left(a_{\ell}+c_{1}\right)} e^{-c_{1} x}+O\left(e^{-c_{2} x}+e^{-\left(c_{1}+1\right) x}\right) .
$$

We replace the doubly indexed family of powers by a singly indexed sequence by introducing $j=m k+r$ and $\lambda_{j}=a_{r+1}+k$ and $\eta_{j}=c_{r+1}+k$, thus obtaining the sequences

$$
\left(\lambda_{j}\right)_{j=0}^{\infty}=\left(a_{1}, a_{2}, \ldots, a_{m}, a_{1}+1, a_{2}+1, \ldots, a_{m}+1, a_{1}+2, \ldots\right),
$$




$$
\left(\eta_{j}\right)_{j=0}^{\infty}=\left(c_{1}, c_{2}, \ldots, c_{m}, c_{1}+1, c_{2}+1, \ldots, c_{m}+1, c_{1}+2, \ldots\right),
$$

where there is a recurring pattern of length $m$. With the coefficients given above, suitably re-indexed, let

$$
\phi_{1}(x)=\sum_{j=0}^{\infty} \xi_{j} e^{-\lambda_{j} x}, \quad \phi_{2}(x)=\sum_{j=0}^{\infty} \gamma_{j} e^{-\eta_{j} x} .
$$

Proposition 6.1. Suppose that $\phi_{1}$ and $\phi_{2}$ are as in (6.8), (6.9) and (6.10). Then the determinant from Corollary 5.2 is given by

$$
\left.\operatorname{det}\left(I-\Gamma_{\phi_{1}} \Gamma_{\phi_{2}}\right)\right|_{L^{2}(x, \infty)}=\operatorname{det}\left[\begin{array}{cc}
I \\
{\left[\frac{e^{-x\left(\eta_{j}+\lambda_{\ell}\right)}}{\eta_{j}+\lambda_{\ell}} \gamma_{j, \ell=0}^{\infty}\right.} & {\left[\frac{e^{-x\left(\eta_{\ell}+\lambda_{j}\right)}}{\eta_{\ell}+\lambda_{j}}\right]_{j, \ell=0}^{\infty}} \\
I
\end{array}\right] .
$$

Proof. We have a series of rank-one kernels

$$
\Gamma_{\phi_{1, x}} \leftrightarrow \sum_{j=0}^{\infty} \xi_{j} e^{-2 \lambda_{j} x} e^{-\lambda_{j}(s+t)},
$$

where $\sum\left|\xi_{j}\right| e^{-\lambda_{j} x} /\left|\lambda_{j}\right|$ converges, so $\Gamma_{\phi_{1}}$ is trace class on $L^{2}(x, \infty)$. Then we introduce the linear systems $\left(-A_{1}, B_{1}, C_{1}\right)$ with $\mathcal{D}\left(A_{1}\right)=\mathcal{D}\left(A_{2}\right)=\left\{\left(u_{j}\right)_{j=0}^{\infty} \in \ell^{2}:\left(j u_{j}\right)_{j=0}^{\infty} \in \ell^{2}\right\}$.

$$
\begin{aligned}
A_{1}: \mathcal{D}\left(A_{1}\right) \rightarrow \ell^{2}: & \left(u_{j}\right)_{j=0}^{\infty} \mapsto\left(\lambda_{j} u_{j}\right)_{j=0}^{\infty} ; \\
B_{1}: \mathbb{C} \rightarrow \ell^{2}: & \beta \mapsto\left(\xi_{j}\right)_{j=0}^{\infty} \beta ; \\
C_{1}: \mathcal{D}\left(A_{1}\right) \rightarrow \mathbb{C}: & \left(u_{j}\right) \mapsto \sum_{j=0}^{\infty} u_{j} ;
\end{aligned}
$$

and likewise $\left(-A_{2}, B_{2}, C_{2}\right)$

$$
\begin{aligned}
A_{2}: \mathcal{D}\left(A_{2}\right) \rightarrow \ell^{2}: & \left(u_{j}\right)_{j=0}^{\infty} \mapsto\left(\eta_{j} u_{j}\right)_{j=0}^{\infty} ; \\
B_{2}: \mathbb{C} \rightarrow \ell^{2}: & \beta \mapsto\left(\gamma_{j}\right)_{j=0}^{\infty} \beta ; \\
C_{2}: \mathcal{D}\left(A_{2}\right) \rightarrow \mathbb{C}: & \left(u_{j}\right) \mapsto \sum_{j=0}^{\infty} u_{j} ;
\end{aligned}
$$

when we combine them into $(-A, B, C)$

$$
\left(\left[\begin{array}{cc}
-A_{1} & 0 \\
0 & -A_{2}
\end{array}\right],\left[\begin{array}{cc}
B_{1} & 0 \\
0 & B_{2}
\end{array}\right],\left[\begin{array}{cc}
0 & C_{2} \\
C_{1} & 0
\end{array}\right]\right)
$$

with scattering function

$$
\begin{aligned}
\Phi(x) & =C e^{-\chi A} B \\
& =\left[\begin{array}{cc}
0 & C_{2} e^{-\chi A_{2}} B_{2} \\
C_{1} e^{-x A_{1}} B_{1} & 0
\end{array}\right] \\
& =\left[\begin{array}{cc}
0 & \phi_{2}(x) \\
\phi_{1}(x) & 0
\end{array}\right],
\end{aligned}
$$


and write $\Phi(t+2 x)=\Phi_{(x)}(t)$. We also consider the operator

$$
\begin{aligned}
R_{X} & =\int_{x}^{\infty} e^{-t A} B C e^{-t A} d t \\
& =\int_{x}^{\infty}\left[\begin{array}{cc}
e^{-A_{1} t} & 0 \\
0 & e^{-A_{2} t}
\end{array}\right]\left[\begin{array}{cc}
B_{1} & 0 \\
0 & B_{2}
\end{array}\right]\left[\begin{array}{cc}
0 & C_{2} \\
C_{1} & 0
\end{array}\right]\left[\begin{array}{cc}
e^{-A_{1} t} & 0 \\
0 & e^{-A_{2} t}
\end{array}\right] d t \\
& =\left[\begin{array}{cc}
0 & \int_{x}^{\infty} e^{-A_{1} t} B_{1} C_{2} e^{-A_{2} t} d t \\
\int_{x}^{\infty} e^{-A_{2} t} B_{2} C_{1} e^{-t A_{1}} d t & 0
\end{array}\right] .
\end{aligned}
$$

To help compute the Fredholm determinant of $R_{x}$, we also let $\Xi_{x}: L^{2}(0, \infty) \rightarrow \ell^{2}$ and $\Theta_{x}: L^{2}(0, \infty) \rightarrow \ell^{2}$ be defined by

$$
\begin{gathered}
\Xi_{x} f=\int_{x}^{\infty} e^{-t A} B f(t) d t, \\
\Theta_{x} f=\int_{x}^{\infty} e^{-s A^{\dagger}} C^{\dagger} f(s) d s .
\end{gathered}
$$

We observe that $\Theta_{x}$ is trace class, and likewise $\Xi_{x}$ is trace class since $\sum_{j} \xi_{j} e^{-\lambda_{j} x}$ converges absolutely. Whereas $\left(e^{-\lambda_{j} t}\right)_{j=0}^{\infty}$ is not an orthogonal basis, the map $\Theta_{x}$ is injective by Lerch's uniqueness theorem for the Laplace transform and $\operatorname{span}\left\{e^{-\lambda_{j} t}, j=0,1, \ldots\right\}$ is dense in $L^{2}(0, \infty)$.

Then we observe that $\Gamma_{\Phi_{(x)}}=\Theta_{x}^{\dagger} \Xi_{x}: L^{2}(0, \infty) \rightarrow L^{2}(0, \infty)$ and $R_{X}=\Xi_{x} \Theta_{x}^{\dagger}: \ell^{2} \rightarrow \ell^{2}$, so that

$$
\operatorname{det}\left(I+R_{\chi}\right)=\operatorname{det}\left(I+\Xi_{\chi} \Theta_{\chi}^{\dagger}\right)=\operatorname{det}\left(I+\Theta_{x}^{\dagger} \Xi_{X}\right)=\operatorname{det}\left(I+\Gamma_{\Phi_{(x)}}\right) .
$$

hence

$$
\operatorname{det}\left(I+R_{X}\right)=\left.\operatorname{det}\left(I-\Gamma_{\phi_{1}} \Gamma_{\phi_{2}}\right)\right|_{L^{2}(0, \infty)} .
$$

With respect to the standard orthonormal basis of $\ell^{2}$, we have a matrix representation

$$
R^{1,2}=\int_{x}^{\infty} e^{-A_{1} t} B_{1} C_{2} e^{-A_{2} t} d t=\left[\frac{e^{-x\left(\eta_{\ell}+\lambda_{j}\right)} \xi_{j}}{\eta_{\ell}+\lambda_{j}}\right]_{j, \ell=0}^{\infty}
$$

for the top right corner of $R_{X}$ as in (6.17), and

$$
R^{2,1}=\int_{x}^{\infty} e^{-A_{2} t} B_{2} C_{1} e^{-A_{1} t} d t=\left[\frac{e^{-x\left(\eta_{j}+\lambda_{\ell}\right)} \gamma_{j}}{\eta_{j}+\lambda_{\ell}}\right]_{j, \ell=0}^{\infty}
$$

for the bottom left corner of $R_{x}$ as in (6.17).

Whereas $R^{2,1}$ is not quite the transpose of $R^{1,2}$, the matrices have a high degree of symmetry which becomes clear when we make our expansion of the determinant. For a finite subset $S$ of $\mathbb{N} \cup\{0\}$, let $\sharp S$ be the cardinality of $S$, and $\Delta_{j \in S}\left(\lambda_{j}\right)$ be Vandermonde's determinant formed from $\lambda_{j}$ with $j \in S$ naturally ordered. For an infinite matrix $V$, and $T \subset \mathbb{N} \cup\{0\}$, let $\operatorname{det}[V]_{S \times T}$ be the determinant formed from the submatrix of $V$ with rows indexed by $j \in S$ and columns indexed by $\ell \in T$, naturally ordered. Then

$$
\begin{aligned}
\operatorname{det}\left(I-R^{1,2} R^{2,1}\right) & =\sum_{S \subset \mathbb{N} \cup\{0\}}(-1)^{\sharp S} \operatorname{det}\left[R^{1,2} R^{2,1}\right]_{S \times S} \\
& =\sum_{S, T \subset \mathbb{N} \cup\{0\}, \sharp S=\sharp T}(-1)^{\sharp S} \operatorname{det}\left[R^{1,2}\right]_{S \times T} \operatorname{det}\left[R^{2,1}\right]_{T \times S},
\end{aligned}
$$


by the Cauchy-Binet formula. Then by Cauchy's formula, the summand involving $S \times T$ is

$$
\begin{aligned}
\operatorname{det}\left[R^{1,2}\right]_{S \times T} \operatorname{det}\left[R^{2,1}\right]_{T \times S}= & \exp \left(-x \sum_{j \in S} \lambda_{j}-x \sum_{\ell \in T} \eta_{\ell}\right) \frac{\prod_{j \in S} \xi_{j} \Delta_{j \in S}\left(\lambda_{j}\right) \Delta_{\ell \in T}\left(\eta_{\ell}\right)}{\prod_{j \in S, \ell \in T}\left(\lambda_{j}+\eta_{\ell}\right)} \\
& \times \exp \left(-x \sum_{j \in T} \eta_{j}-x \sum_{\ell \in S} \lambda_{\ell}\right) \frac{\prod_{j \in T} \gamma_{j} \Delta_{j \in T}\left(\eta_{j}\right) \Delta_{\ell \in S}\left(\lambda_{\ell}\right)}{\prod_{\ell \in S, j \in T}\left(\lambda_{\ell}+\eta_{j}\right)} .
\end{aligned}
$$

Hence we have the determinant expansion of

$$
\operatorname{det}\left(I-R^{1,2} R^{2,1}\right) . \quad=\sum_{S, T \subset \mathbf{N} \cup\{0\}, \sharp S=\sharp T}(-1)^{\sharp S} \exp \left(-2 x \sum_{j \in S} \lambda_{j}-2 x \sum_{\ell \in T} \eta_{\ell}\right) \frac{\prod_{j \in S} \xi_{j} \prod_{\ell \in T} \gamma_{\ell} \Delta_{j \in S}\left(\lambda_{j}\right)^{2} \Delta_{\ell \in T}\left(\eta_{\ell}\right)^{2}}{\prod_{j \in S, \ell \in T}\left(\lambda_{j}+\eta_{\ell}\right)^{2}} .
$$

Formulas such as (6.25) appear in applications of representation theory to solitons, as in [20, p. 234]. We now make an approximation, similar to [2, (2.30)]. Suppose that $x$ is large, so that we only need retain the largest terms, which arise from $j=\ell=0$, that is $S=T=\{0\}$; then

$$
\begin{aligned}
\operatorname{det}\left(I+R_{X}\right) & =\operatorname{det}\left[\begin{array}{cc}
1 & \frac{e^{-x\left(\eta_{0}+\lambda_{0}\right)} \xi_{0}}{\eta_{0}+\lambda_{0}} \\
\frac{e^{-x\left(\eta_{0}+\lambda_{0}\right)} \gamma_{0}}{\eta_{0}+\lambda_{0}} & 1
\end{array}\right] \\
& =1-\frac{e^{-2 x\left(\lambda_{0}+\eta_{0}\right)} \xi_{0} \gamma_{0}}{\left(\eta_{0}+\lambda_{0}\right)^{2}} .
\end{aligned}
$$

Definition 6.2. For $\Omega$ a domain in $\mathbb{C}$, a divisor is function $\delta: \Omega \rightarrow \mathbb{Z}$ such that $\{z: \delta(z) \neq 0\}$ has no limit points in $\Omega$. In particular, the function $\delta_{z}: \mathbb{C} \rightarrow \mathbb{Z}$ given by $\delta_{z}(x)=1$ for $x=z$ and $\delta_{z}(x)=0$ for $x \neq z$ is a divisor.

The set of all divisors on $\Omega$ forms an additive group $\mathcal{D}(\Omega)$. For each meromorphic function, we associate the divisor given by the sum of $n \delta_{z}$ for each zero of order $n$ at $z$, and $-m \delta_{p}$ for each pole of order $m$ at $p$. For $\Gamma$-functions, it is convenient to have the following shorthand. For $s \in \mathbb{C}$ we write, with a subscript $R$ denoting an arithmetic progression to the right,

$$
(s)_{R}=\delta_{s}+\delta_{s+1}+\delta_{s+2}+\ldots,
$$

and, with a subscript $L$ denoting an arithmetic progression to the left,

$$
(s)_{L}=\delta_{s}+\delta_{s-1}+\delta_{s-2}+\ldots
$$

There is an additive subgroup $\mathcal{D}_{\Gamma}$ of $\mathcal{D}(\mathbb{C})$ generated by the $\pm(a)_{L}$ and $\pm(b)_{R}$ with $a, b \in \mathbb{C}$, so that every $\delta \in \mathcal{D}_{\Gamma}$ arises from a quotient of products of Gamma functions, and $\mathcal{D}_{\Gamma}$ contains all finitely supported divisors.

\section{Examples}

Example 7.1. (i) Suppose that $\sum_{j=1}^{m}\left(-a_{j}+b_{j}\right)+\sum_{j=1}^{\mu}\left(-c_{j}+d_{j}\right)=0$. Then there exists $\lambda$ such that $1-\lambda+\sum_{j=1}^{m}\left(-a_{j}+\right.$ $\left.b_{j}\right)=0$ and $1-\lambda+\sum_{j=1}^{\mu}\left(c_{j}-d_{j}\right)=0$. Hence we can apply Corollary 5.2 to $S(\xi \mid \lambda)=\psi(i \xi)=\tilde{\psi}_{-}(i \xi) \tilde{\psi}_{+}(i \xi)$ for the Wiener-Hopf factors

$$
\tilde{\psi}_{-}(i \xi)=\frac{\Gamma(\lambda-i \xi)}{\Gamma(1-i \xi)} \prod_{j=1}^{m} \frac{\Gamma\left(a_{j}+i \xi\right)}{\Gamma\left(b_{j}+i \xi\right)}, \quad \tilde{\psi}_{+}(i \xi)=\frac{\Gamma(1+i \xi)}{\Gamma(\lambda+i \xi)} \prod_{j=1}^{\mu} \frac{\Gamma\left(c_{j}-i \xi\right)}{\Gamma\left(d_{j}-i \xi\right)} .
$$


(ii) This example arises via the scattering amplitude in one-dimensional scattering theory. Let $q \in C_{c}^{\infty}(\mathbb{R} ; \mathbb{R})$, and consider the Schrödinger equation with even potential $q$. There exist an even solution $f_{+}$and an odd solution $f_{\text {- such that }}$

$$
-\frac{d^{2} f_{ \pm}}{d x^{2}}(x ; \xi)+q(x) f_{ \pm}(x ; \xi)=\xi^{2} f_{ \pm}(x ; \xi)
$$

such that

$$
\begin{aligned}
& f_{ \pm}(x ; \xi) \asymp e^{-i \xi x}-e^{i \xi x-i \theta_{ \pm}(\xi)} \quad(x \rightarrow \infty) \\
& f_{ \pm}(x ; \xi) \asymp \pm\left(e^{-i \xi x}-e^{i \xi x-i \theta_{ \pm}(\xi)}\right) \quad(x \rightarrow-\infty)
\end{aligned}
$$

so $\theta_{ \pm}$is the phase shift. Let the reflection coefficient be $R(\xi)=(-1 / 2)\left(e^{-i \theta_{+}(\xi)}+e^{-i \theta_{-}(\xi)}\right)$. Then with $\phi(x)=$ $(2 \pi)^{-1} \int_{-\infty}^{\infty} e^{i \xi x} R(\xi) d \xi$, we introduce $\Gamma_{\phi}$ and

$$
\vartheta=\operatorname{det}\left(I+\Gamma_{\phi}\right)
$$

as in [11]. In [11, section 5.7], the authors interpret $\vartheta$ as a theta function on an infinite-dimensional torus, and obtain series expansions for the determinant. In the current paper, we use the exponential series (2.13) and (6.12) instead, which lead to formulas (6.25) which resemble those on [11, 5.7, 5.8].

In particular, consider the Schrödinger equation as in [34, p. 36]

$$
-f^{\prime \prime}(x)+\frac{\lambda(\lambda-1)}{\sinh ^{2} x} f(x)=\xi^{2} f(x) .
$$

Then the scattering amplitude is the coefficient of $f(x)$ for large $x$ when $e^{-i \lambda x}$ is the scattering function. Then

$$
S(\xi \mid \lambda)=-e^{-i \theta_{+}(\xi)}=-\frac{\Gamma(1+i \xi) \Gamma(\lambda-i \xi)}{\Gamma(1-i \xi) \Gamma(\lambda+i \xi)}
$$

which has divisor, in terms of $s=i \xi$,

$$
-(-1)_{L}-(-\lambda)_{R}+(1)_{R}+(-\lambda)_{L}
$$

Example 7.2. Meier's $G$-function [12, p. 206] is

$$
G_{p, q}^{m, n}\left(x \mid \begin{array}{l}
a_{1}, \ldots, a_{p} \\
b_{1}, \ldots, b_{q}
\end{array}\right)=\frac{1}{2 \pi i} \int_{\gamma-i \infty}^{\gamma+i \infty} \frac{\prod_{j=1}^{m} \Gamma\left(b_{j}-s\right) \prod_{j=1}^{n} \Gamma\left(1-a_{j}+s\right)}{\prod_{j=m+1}^{q} \Gamma\left(1-b_{j}+s\right) \prod_{j=n+1}^{p} \Gamma\left(a_{j}-s\right)} x^{s} d s,
$$

where we take all the $a_{j}, b_{j}$ in $\{s: 0<\operatorname{Re} s<1\}$ with degree $p+q-2 m-2 n$, which we take to be negative. Then the divisor for the quotient of Gamma functions in the integrand is

$$
\sum_{j=1}^{m}-\left(b_{j}\right)_{R}-\sum_{j=1}^{n}\left(a_{j}-1\right)_{L}+\sum_{j=m+1}^{q}\left(b_{j}-1\right)_{L}+\sum_{j=n+1}^{p}\left(a_{j}\right)_{R} .
$$

Then the integral converges for $|\arg x|<(2 m+2 n-p-q) \pi / 2$.

One can express various applications of Corollary 5.3 in terms of $G$.

Example 7.3. Hankel matrices also arise from functions on the finite-dimensional real torus. Let $1 / 2<v<1$ and observe that Struve's function $S_{v}$ [33, p. 127] has Mellin transform

$$
S_{v}^{\star}(s)=2^{s-1} \frac{\tan ((\pi / 2)(s+v)) \Gamma((s+v) / 2)}{\Gamma((v-s+2) / 2)},
$$

which is holomorphic on $-v<\operatorname{Re} s<1-v$; see [30]. Also, for $s=\eta+i \xi$ and $-1<\eta<-1 / 2$, we have $S_{\nu}^{\star}(\eta+i \xi)=O\left(|\xi|^{\eta}\right)$ as $\xi \rightarrow \pm \infty$, so $S^{\star}(\eta+i \xi) \rightarrow 0$ as $\xi \rightarrow \pm \infty$ and

$$
\int_{-\infty}^{\infty}\left|S_{v}^{\star}(\eta+i \xi)\right|^{2} d \xi<\infty
$$


hence by Plancherel's formula, we have

$$
\int_{0}^{\infty} x^{2 \eta-1} S_{v}(x)^{2} d x<\infty
$$

We have the determinant of the finite Hankel matrix

$$
\begin{aligned}
I_{n}(t) & =\frac{\pi^{n^{2} / 2}}{t^{n(n-1)} 2^{n(n-1)}} \operatorname{det}\left[\Gamma(j+k+1 / 2) S_{j+k}(t)\right]_{j, k=0, \ldots, n-1} \\
& =\operatorname{det}\left[\frac{S_{j+k}(t) \Gamma(j+k+1 / 2) \sqrt{\pi}}{2^{j+k} t^{j+k}}\right]_{j, k=0, \ldots, n-1} \\
& =\operatorname{det}\left[\int_{[0, \pi / 2]} \sin ^{2(j+\ell)} \theta \sin (t \cos \theta) d \theta\right]_{j, k=0, \ldots, n-1} \\
& =\frac{1}{n !} \int_{[0, \pi / 2]^{n}} \operatorname{det}\left[\sin ^{2 k} \theta_{j}\right]_{j, k=0, \ldots, n-1}^{2} \prod_{j=0}^{n-1} \sin \left(t \cos \theta_{j}\right) d \theta_{j} \\
& =\frac{1}{n !} \int_{[0, \pi / 2]^{n}} \prod_{j, k=0, \ldots, n-1 ; j<k}\left(\sin ^{2} \theta_{j}-\sin ^{2} \theta_{k}\right)^{2} \prod_{j=0}^{n-1} \sin \left(t \cos \theta_{j}\right) d \theta_{j},
\end{aligned}
$$

where the final formula resembles the Weyl integration formula for a class function on the symplectic group $S p(n)$.

The following example gives a case in which moments satisfy a type of recurrence relation, but do not quite satisfy the conclusions of the Theorem (3.2). The linear system representation is found explicitly.

Proposition 7.4. For $\kappa>1$, introduce the weight $w(x)=\log (2 \kappa /(1-x))$ for $-1<x<1$. Then the moment matrix $\left[\mu_{j+k}\right]_{j, k=0}^{\infty}$ defines a bounded linear operator on $\ell^{2}$ which is not Hilbert-Schmidt.

Proof. Here $w(x) \rightarrow \infty$ as $x \rightarrow 1-$, and $w(x) \rightarrow \log \kappa$ as $x \rightarrow(-1)+$. The moments satisfy

$$
\mu_{0}=\int_{-1}^{1} w(x) d x=2 \log (2 \kappa)+2-2 \log 2
$$

and generally

$$
\mu_{n}=\int_{-1}^{1} x^{n} w(x) d x=\log (2 \kappa) \int_{-1}^{1} x^{n} d x-\int_{-1}^{1} x^{n} \log (1-x) d x,
$$

where by integrating by parts, one obtains

$$
\begin{aligned}
\int_{-1}^{1} & -x^{n} \log (1-x) d x \\
& =\left[x^{n}(1-x) \log (1-x)\right]_{-1}^{1}+\int_{-1}^{1} x^{n} d x-n \int_{-1}^{1} x^{n-1}(1-x) \log (1-x) d x \\
& =-(-1)^{n} 2 \log 2+\frac{1-(-1)^{n+1}}{n+1}-n \int_{-1}^{n} x^{n-1} \log (1-x) d x+n \int_{-1}^{1} x^{n} \log (1-x) d x,
\end{aligned}
$$

so by solving the recurrence relation

$$
-(n+1) \int_{-1}^{1} x^{n} \log (1-x) d x=-n \int_{-1}^{1} x^{n-1} \log (1-x) d x-(-1)^{n} 2 \log 2+\frac{1-(-1)^{n+1}}{n},
$$


we obtain

$$
-(n+1) \int_{-1}^{1} x^{n} \log (1-x) d x=-\int_{-1}^{1} \log (1-x) d x-2 \log 2 \sum_{m=1}^{n}(-1)^{m}+\sum_{m=1}^{n} \frac{1-(-1)^{m+1}}{m+1}
$$

hence

$$
\begin{aligned}
\int_{-1}^{1} x^{n} w(x) d x= & \frac{1-(-1)^{n+1}}{n+1} \log (2 \kappa) \\
& +\frac{1}{n+1}\left(2-2 \log 2+\left(1+(-1)^{n+1}\right) \log 2+\sum_{m=1}^{n} \frac{1-(-1)^{m+1}}{m+1}\right) ;
\end{aligned}
$$

the final term includes the sum

$$
\sum_{m=1}^{n} \frac{1-(-1)^{m+1}}{m+1}=\log n+\log 2+\gamma+o(1) \quad(n \rightarrow \infty),
$$

where here $\gamma$ is Euler's constant. Hence the Cauchy transform

$$
G(z)=\int_{-1}^{1} \frac{w(t) d t}{z-t}=\sum_{n=0}^{\infty} \frac{\mu_{n}}{z^{n+1}}
$$

diverges at some points with $|z|=1$. Also $\sum_{j=0}^{\infty} j \mu_{j}^{2}$ diverges, so the Hankel moment matrix is not HilbertSchmidt.

Nevertheless, the Hankel moment matrix $\left[\mu_{j+m}\right]_{j, m=0}^{\infty}$ defines a bounded linear operator on $\ell^{2}$. To see this, we transform to Hankel integral operators on $L^{2}(0, \infty)$ via the Laguerre functions. With $J_{0}$ standing for Bessel's function of the first kind of order zero, the orthonormal Laguerre functions in $L^{2}(0, \infty)$ satisfy

$$
e^{-x / 2} L_{n}(x)=\frac{e^{x / 2}}{n !} \int_{0}^{\infty} t^{n} J_{0}(2 \sqrt{x t}) e^{-t} d t .
$$

We introduce the scattering function

$$
\phi(x)=\sum_{n=0}^{\infty} \mu_{n} e^{-x / 2} L_{n}(x)
$$

which we can express as an integral

$$
\begin{aligned}
\phi(x) & =e^{x / 2} \sum_{n=0}^{\infty} \int_{0}^{\infty} \frac{t^{n} \mu_{n}}{n !} J_{0}(2 \sqrt{x t}) e^{-t} d t \\
& =e^{x / 2} \sum_{n=0}^{\infty} \int_{0}^{\infty} \int_{-1}^{1} v^{n} w(v) d v \frac{t^{n}}{n !} J_{0}(2 \sqrt{x t}) e^{-t} d t \\
& =e^{x / 2} \int_{0}^{\infty} \int_{-1}^{1} \sum_{n=0}^{\infty} v^{n} \frac{t^{n}}{n !} w(v) d v J_{0}(2 \sqrt{x t}) e^{-t} d t \\
& =e^{x / 2} \int_{-1}^{1} \int_{0}^{\infty} e^{v t} J_{0}(2 \sqrt{x t}) e^{-t} d t w(v) d v .
\end{aligned}
$$

By Webber's integral [13, 4.14(25), p. 185], the inside integral is

$$
\int_{0}^{\infty} e^{-t+v t} J_{0}(2 \sqrt{x t}) d t=\frac{1}{1-v} e^{-x /(1-v)},
$$


so we have, on substituting $s=1 /(1-v)$,

$$
\begin{aligned}
\phi(x) & =e^{x / 2} \int_{-1}^{1} w(v) e^{-x /(1-v)} \frac{d v}{1-v} \\
& =e^{x / 2} \int_{1 / 2}^{\infty} \log (2 \kappa s) e^{-s x} \frac{d s}{s} .
\end{aligned}
$$

It is convenient to introduce the incomplete Gamma function

$$
\phi_{v}(x)=e^{x / 2} \int_{1 / 2}^{\infty} s^{v-1} e^{-x s} d s
$$

and write

$$
\phi(x)=\log (2 \kappa) \phi_{0}(x)+\left(\frac{\partial}{\partial v}\right)_{v=0} \phi_{v}(x) .
$$

We now express $\phi_{v}$ as the scattering function of a continuous time linear system. Let the state space be $H=L^{2}(0, \infty)$, with dense linear subspace $\mathcal{D}(A)=\{f \in H: t f(t) \in H\}$. Then for $-1 / 2 \leq \operatorname{Re} v<1 / 2$, we introduce the linear system $\left(-A, B_{v}, C\right)$ by

$$
\begin{array}{rlrl}
A: \mathcal{D}(A) \rightarrow H: & f & \mapsto t f(t) \quad(f \in \mathcal{D}(A)) \\
B_{v}: \mathbb{C} \rightarrow H: & b & \mapsto b(1 / 2+t)^{v-1} b \quad(b \in \mathbb{C}) \\
C: \mathcal{D}(A) \rightarrow \mathbb{C}: & f \mapsto \int_{0}^{\infty} f(t) d t \quad(f \in \mathcal{D}(A)) ;
\end{array}
$$

the corresponding scattering function is

$$
\phi_{v}(x)=C e^{-x A} B_{v}=\int_{0}^{\infty}(1 / 2+t)^{v-1} e^{-x t} d t .
$$

Then we introduce the operator

$$
R_{v}=\int_{0}^{\infty} e^{-x A} B_{v} C e^{-x A} d x
$$

which is the integral operator on $L^{2}(0, \infty)$ that has kernel

$$
R_{v}(\tau, t)=\frac{(1 / 2+\tau)^{v}}{(1 / 2+\tau)} \frac{1}{t+\tau}
$$

Evidently $R_{v}$ is the composition of Hilbert's Hankel operator with kernel $1 /(\tau+t)$ and multiplication by $(1 / 2+$ $\tau)^{v-1}$, so $R_{v}$ is bounded on $L^{2}(0, \infty)$. Operators of this form were considered by Howland [18].

\section{Application of equilibrium problem to linear statistics}

Let $M_{n}^{h}(\mathbb{C})$ be the space of $n \times n$ complex Hermitian matrices, and $d X$ Lebesgue measure on the entries on or above the leading diagonal. Let $v_{0}: \mathbb{R} \rightarrow \mathbb{R}$ be a twice continuously differentiable function and $M>0$ such that $v_{0}(x) \geq 2 \log \left(1+x^{2}\right)$ for all $x \in \mathbb{R}$ such that $|x|>M$. The eigenvalues of $X_{n} \in M_{n}(\mathbb{C})$ are listed as $\lambda_{1} \leq \lambda_{2} \leq \cdots \leq \lambda_{n}$ according to multiplicity. Choosing

$$
Z_{n}=\int_{\mathbb{R}^{n}} e^{-n \sum_{j=1}^{n} v_{0}\left(\lambda_{j}\right)} \prod_{1 \leq j<k \leq n}\left(\lambda_{j}-\lambda_{k}\right)^{2} d \lambda_{1}, \ldots d \lambda_{N}
$$


we obtain a probability measure

$$
v_{n}(d X)=Z_{n}^{-1} \exp \left(-n \operatorname{trace} v_{0}(X)\right) d X
$$

on $M_{n}^{h}(\mathbb{C})$ that is invariant under the unitary conjugation $X \mapsto U X U^{\dagger}$ for $X \in M_{n}^{h}(\mathbb{C})$. On the probability space $\left(M_{n}^{h}(\mathbb{C}), v_{n}\right)$, for each bounded measurable $f: \mathbb{R} \rightarrow \mathbb{C}$, we consider the distribution of the linear eigenvalue statistic trace $f(X)=\sum_{j=1}^{n} f\left(x_{j}\right)$, which captures information about the equilibrium measure associated with $v_{0}$.

In this section we consider

$$
\mathbb{E} e^{-\sum f}=\frac{\int_{(0, b)^{n}} \exp \left(-\sum_{j=1}^{n} f\left(x_{j}\right)\right) \prod_{1 \leq j<k \leq n}\left(x_{j}-x_{k}\right)^{2} \prod_{j=1}^{n} w_{0}\left(x_{j}\right) d x_{j}}{\int_{(0, b)^{n}} \prod_{1 \leq j<k \leq n}\left(x_{j}-x_{k}\right)^{2} \prod_{j=1}^{n} w_{0}\left(x_{j}\right) d x_{j}},
$$

with particular emphasis on $w_{0}=e^{-n v_{0}}$ where $v_{0} \in C^{2}$ is convex and $f(x)=9(x-t)$ is a step function. Then the exponent in numerator of the expression (1.3) involves

$$
\frac{1}{n^{2}} \sum_{j=1}^{n} f\left(x_{j}\right)+\frac{1}{n} \sum_{j=1}^{n} v_{0}\left(x_{j}\right)-\frac{1}{n^{2}} \sum_{1 \leq j, k \leq n: j \neq k} \log \left|x_{j}-x_{k}\right| .
$$

We regard this as the electrostatic energy associated with $n$ positive and equal charges on a line, subject to an electrical field. The following result [31] extends a familiar result to the case of discontinuous fields.

Lemma 8.1. Let $\Sigma$ be a closed subset of the Riemann sphere and let $v: \Sigma \rightarrow \mathbb{R} \cup\{ \pm \infty\}$ be lower semi continuous, $v<\infty$ on a set of positive logarithmic capacity and suppose that there exists $c>0$ such that $v(z) \geq c \log |z|$ as $z \rightarrow \infty$ for $z \in \Sigma$. Then the minimization problem in the collection of all probability measures on $\Sigma$,

$$
\inf _{\sigma}\left\{\int_{\Sigma} v(x) \sigma(d x)+\iint_{\Sigma \times \Sigma} \log \frac{1}{|x-y|} \sigma(d x) \sigma(d y)\right\}
$$

has a unique minimizer $\sigma$, with support $S \subseteq \Sigma$. Furthermore, there exists $C \in \mathbb{R}$ such that

$$
v(x)=2 \int_{S} \log |x-y| \sigma(d y)+C
$$

for quasi almost all $x$ in $S$.

Let $v_{0}$ be $C^{2}$ and convex, with $v_{0}(x) \geq c \log (1 / x)$ as $x \rightarrow 0+$ and $v_{0}(x) \geq c \log x$ as $x \rightarrow \infty$ for some $c>0$. Then there exists a probability measure $\sigma_{0}$ supported on $[a, b] \subset(0, \infty)$ and constant $C$ such that

$$
v_{0}(x) \geq 2 \int_{a}^{b} \log |x-y| \sigma_{0}(d y)+C
$$

with equality for all $x \in(a, b)$. We replace the weight $w_{0}$ by $w$, the potential $v_{0}$ by $v=v_{0}+\beta f$, hence $\sigma_{0}$ by $\sigma=\sigma_{0}+\rho$, where $\int \rho=0$, and consider the integral equation

$$
f(x)=2 \int_{a}^{b} \log |x-y| \rho(y) d y+c_{1} .
$$

The probability density of the linear statistic has mean

$$
S_{2}=\int_{a}^{b} f(x) \sigma_{0}(x) d x
$$

and variance

$$
S_{1}=\frac{1}{4 \pi^{2}} \int_{a}^{b} \int_{a}^{b} \frac{f(x)}{\sqrt{(x-a)(b-x)}} \frac{\partial}{\partial y}\left(\frac{\sqrt{(b-y)(y-a)}}{x-y}\right) f(y) d y d x .
$$

To compute $S_{2}$, one uses Fourier series. 
Lemma 8.2. For $n \in \mathbb{N}$, let $(\pi / 2) a_{n}=\int_{0}^{\pi} v_{0}\left(\frac{a+b}{2}+\frac{b-a}{2} \cos \theta\right) \cos n \theta d \theta$. Suppose that $\left(n a_{n}\right) \in \ell^{2}$, and $f$ is bounded. Then

$$
S_{2}=\int_{0}^{\pi} f\left(\frac{a+b}{2}+\frac{b-a}{2} \cos \theta\right) \frac{d \theta}{\pi}-\sum_{n=1}^{\infty} n a_{n} \int_{0}^{\pi} f\left(\frac{a+b}{2}+\frac{b-a}{2} \cos \theta\right) \cos n \theta d \theta .
$$

Proof. We write

$$
v_{0}\left(\frac{a+b}{2}+\frac{b-a}{2} \cos \theta\right)=\sum_{n=0}^{\infty} a_{n} \cos n \theta
$$

Then for $n \in \mathbb{N}$, we let

$$
h_{n}(\phi)=\int_{0}^{\pi} \log |\cos \phi-\cos \theta| \cos n \theta d \theta
$$

so

$$
h_{n}^{\prime}(\phi)=-\sin \phi \text { p.v. } \int_{0}^{\pi} \frac{\cos n \theta}{\cos \phi-\cos \theta} d \theta=\sin n \phi .
$$

For $n$ even, $h_{n}(\pi / 2)$ is given by

$$
\int_{0}^{\pi} \log |\cos \theta| \cos (2 k \theta) d \theta=2^{-1} \int_{0}^{2 \pi} \log |\cos (x / 2)| \cos k x d x=\frac{(-1)^{k} \pi}{2 k},
$$

whereas for $n$ odd, $h_{n}(\pi / 2)$ is given by

$$
\int_{0}^{\pi} \log |\cos \theta| \cos (2 k+1) \theta d \theta=0
$$

so

$$
h_{n}(\pi / 2)-h_{n}(\phi)=\int_{\phi}^{\pi / 2} \sin n \psi d \psi=-\frac{\cos n \pi / 2}{n}+\frac{\cos n \phi}{n},
$$

so $h_{n}(\phi)=\frac{-1}{n} \cos n \phi$. We can therefore write a solution of the extremal problem as

$$
\sigma_{0}\left(\frac{a+b}{2}+\frac{b-a}{2} \cos \theta\right)|\sin \theta|=\frac{1}{\pi}-\sum_{n=1}^{\infty} n a_{n} \cos n \theta \quad(0<\theta<\pi),
$$

which by hypothesis is $L^{2}$ convergent, and then we establish the equality

$$
\begin{aligned}
S_{2} & =\int_{0}^{\pi} f\left(\frac{a+b}{2}+\frac{b-a}{2} \cos \theta\right) \sigma_{0}\left(\frac{a+b}{2}+\frac{b-a}{2} \cos \theta\right) \sin \theta d \theta \\
& =\int_{0}^{\pi} f\left(\frac{a+b}{2}+\frac{b-a}{2} \cos \theta\right) \frac{d \theta}{\pi}-\sum_{n=1}^{\infty} n a_{n} \int_{0}^{\pi} f\left(\frac{a+b}{2}+\frac{b-a}{2} \cos \theta\right) \cos n \theta d \theta .
\end{aligned}
$$

Example 8.3. (i) In the context of (3.39) Let $[a, b]=[-1,1]$ and for $t \in[-1,1]$ let $f(x)=9(x-t)$, and let $U_{n}$ be the Chebyshev polynomial of the second kind of degree $n$ such that $U_{n}(\cos \phi)=\sin (n+1) \phi / \sin \phi$. Then by the Lemma 8.2, we have

$$
S_{2}=\frac{\cos ^{-1} t}{\pi}-\sum_{n=1}^{\infty} \sqrt{1-t^{2}} U_{n-1}(t)(2 / \pi) \int_{0}^{\pi} v(\cos \theta) \cos n \theta d \theta .
$$


(ii) In the context of (3.31), let $f(x)=\pi^{-1} \tan ^{-1}((x-t) / \varepsilon)$, and consider $-1<t<1$ for $[a, b]=[-1,1]$; with $z_{ \pm}=t \pm i \varepsilon$. Then by considering the integrals

$$
\text { p.v. } \int_{-1}^{1} \frac{\sqrt{1-y^{2}}}{y-x} \frac{d y}{y-z}=-\pi+\frac{\pi \sqrt{z^{2}-1}}{z-x} \quad(z \in \mathbb{C} \backslash[-1,1]),
$$

where the branch of the square root is chosen so that the integrals converge to zero as $z \rightarrow \infty$, we deduce that $\rho$ from (8.4) satisfies

$$
\rho(x)=\frac{\sqrt{1-x^{2}}}{2 \pi^{2}} \frac{1}{2 i}\left(\frac{-1}{\left(x-z_{+}\right) \sqrt{z_{+}^{2}-1}}+\frac{1}{\left(x-z_{-}\right) \sqrt{z_{-}^{2}-1}}\right)+\frac{C}{\sqrt{1-x^{2}}},
$$

where $C$ is chosen so that $\int_{-1}^{1} \rho(x) d x=0$. As we cross $[-1,1]$, the square root changes sign.

Example 8.4. For $f \in L^{\infty}$, we consider

$$
f(x)=\int_{0}^{\infty} \log |x-y| \rho(y) d y+c_{1}
$$

so

$$
f(0)=\int_{0}^{\infty} \log |y| \rho(y) d y+c_{1}
$$

and subtracting, we have a Mellin convolution

$$
f(x)-f(0)=\int_{0}^{\infty} \log \left|\frac{x}{y}-1\right| y \rho(y) \frac{d y}{y},
$$

so

$$
M(f(x)-f(0) ; s)=-\frac{\pi}{s} \tan \pi(s+1 / 2) M(x \rho(x) ; s)
$$

so

$$
M(x \rho(x) ; s)=s^{2}\left(-\frac{\tan \pi s}{\pi s}\right) M(f(x)-f(0) ; s) .
$$

We deduce that

$$
x \rho(x)=\frac{1}{\pi^{2}}\left(x \frac{d}{d x}\right)^{2} \int_{0}^{\infty} y(f(y)-f(0)) \log \frac{\sqrt{x}+\sqrt{y}}{|\sqrt{x}-\sqrt{y}|} \frac{d y}{y} .
$$

Now we let

$$
g_{2}(x)=\left(x \frac{d}{d x}\right)^{2} \log \frac{\sqrt{x}+1}{|\sqrt{x}-1|}=\frac{\sqrt{x}(x+1)}{2(x-1)^{2}}
$$

which has Mellin transform

$$
M\left(g_{2} ; s\right)=\frac{-s \tan \pi s}{\pi} .
$$

Hence by the Plancherel formula for the Mellin transform

$$
\begin{aligned}
\int_{0}^{\infty} x f(x)\left(g_{2} \star f\right)(x) d x & =\frac{1}{2 \pi i} \int_{-i \infty}^{i \infty} M\left(g_{2}, s\right) M(f ; s) M(f ;-s) d s \\
& =\frac{1}{2 \pi^{2}} \int_{-\infty}^{\infty} \xi \tanh (\pi \xi) M(f ; i \xi) M(f ;-i \xi) d \xi .
\end{aligned}
$$

One compares this formula with Proposition 2.2.

Acknowledgments Gordon Blower acknowledges the generous support of a Distinguished Visiting Scholarship at the University of Macau. Yang Chen gratefully acknowledges the generous support of the Macau Science and Technology Development Fund under the grant numbers FDCT 130/2014/ A3 and FDCT 023/2017/A1 and the University of Macau through MYRG2014-00011-FST and MYRG2014-0004-FST. The authors thank the referee for carefully reading the manuscript and helpfully suggesting improvements. 


\section{References}

[1] E.L. Basor, Distribution functions for random variables for ensembles of positive Hermitian matrices, Comm. Math. Phys. 188 (1997), 327-350.

[2] E.L. Basor and Y. Chen, A note on Wiener-Hopf determinants and the Borodin-Okounkov identity, Integral Equations and Operator Theory, 45 (2003), 301-308.

[3] E.L. Basor and Y. Chen, Toeplitz determinants from compatibility conditions, Ramanujan Math. J. 16 (2008), 25-40.

[4] E.L. Basor, Y. Chen and H. Widom, Hankel determinants and Fredholm determinants, pp 21-29, Random matrix models and their applications, (MSRI Publications, Cambridge University Press, 2001).

[5] E.L. Basor and T. Ehrhardt, Asymptotics of determinants of Bessel operators, Comm. Math. Phys. 234 (2003), 491-516.

[6] E.L. Basor and C.A. Tracy, Variance calculations and the Bessel kernel, J. Statistical Physics 73 (1993), 415-421.

[7] G. Blower, On linear systems and $\tau$-functions associated with Lamé's equation and Painlevé's equation VI, J. Math. Anal. Appl. 376 (2011), 294-316.

[8] Y. Chen and A.R. Its, A Riemann-Hilbert approach to the Akhiezer polynomials, Phil. Trans. R. Soc. A 366 (2008), $973-1003$.

[9] Y. Chen and N. Lawrence, On the linear statistics of Hermitian random matrices, J. Phys. A 31 (1998), 1141-1152.

[10] R.G. Douglas, Banach algebra techniques in operator theory, Second edition, (Springer, 1998).

[11] N. Ercolani and H.P. McKean, Geometry of KDV(4): Abel sums, Jacobi variety and theta functions in the scattering case, Invent. Math. 99 (1990), 483-544.

[12] A. Erdelyi, Higher Transcendental Functions, Volume 1, (McGraw, 1953).

[13] A. Erdelyi, Integral Transforms, Volume 1, (McGraw, 1954).

[14] A.S. Fokas, A.R. Its, A.A. Kapaev and V.Y. Novokshenov, Painlevé Transcendents. The Riemann-Hilbert approach. (American Mathematical Society, 2006).

[15] E. Heine, Handbuch der Kugelfunctionen: Theorie und Anwendungen, (Physica Verlag, Würzburg, 1961)

[16] J.W. Helton and R.E. Howe, Integral operators, commutators, traces, index and homology, pp 141-209 in Lecture Notes in Mathematics 345, (Springer, 1973).

[17] N. Hitchin, A lecture on the octahedron, Bull. London Math. Soc 35 (2003), 577-600.

[18] J.S. Howland, Spectral theory of operators of Hankel type II, Indiana Univ. Math. J. 41 (1992), 427-434.

[19] M. Jimbo and T. Miwa, Monodromy preserving deformation of linear ordinary differential equations with rational coefficients. II. Physica D 2 (1981), 407-448.

[20] V.G. Kac, Infinite dimensional Lie algebras, Second Edition, (Cambridge University Press, 1985).

[21] A. Katavolos and S.C. Power, Translation and dilation invariant subspaces of $L^{2}(\mathbb{R})$, J. reine angew. Math. 552 (2002), $101-129$.

[22] A.V. Kitaev and D.A. Korotkin, Solutions of the Schlesinger equations in terms of $\Theta$-functions, Internat. Math. Res. Notices 17 (1998), 877-905.

[23] P. Koosis, Introduction to $H_{p}$ spaces, (Cambridge University Press, 1980).

[24] A.P. Magnus, Painlevé type differential equations for the recurrence coefficients of semi-classical orthogonal polynomials, J. Comput. Appl. Math. 57 (1995), 215-237.

[25] A.V. Megretskii, V.V. Peller, and S.R. Treil, The inverse spectral problem for self-adjoint Hankel operators, Acta Math. 174 (1995), 241-309.

[26] C. Min and Y. Chen, Gap probability distribution of the Jacobi unitary ensemble: elementary treatment, from finite $n$ to double scaling, Stud. Appl. Math. 140 (2018), 202-220.

[27] J. Palmer, Deformation analysis of matrix models, Physica D 78 (1994), 166-185.

[28] J.R. Partington and G. Weiss, Admissible observation operators for the right-shift semigroup, Math. Control Signals Systems, 13 (2000), 179-192.

[29] V.V. Peller, Hankel Operators and their Applications, (Springer, 2003).

[30] M. van der Put and M.F. Singer, Galois theory of linear differential equations, (Springer, 2003).

[31] P. Simeonov, A weighted energy problem for a class of admissible weights, Houston J. Math. 31 (2005), 1245-1260.

[32] V.I. Smirnov, Integral Equations and Partial Differential Equations, volume IV, (Pergamon, 1964).

[33] I.N. Sneddon, The use of integral transforms, (McGraw-Hill, 1972).

[34] B. Sutherland, Beautiful models: 70 years of exactly solved quantum many-body problems, (World Scientific, 2004).

[35] J.L. Taylor, Banach algebras and topology pp 118-186 in Algebras in Analysis J.R. Williamson (edr), (Academic Press, 1975).

[36] J.L. Taylor, Topological invariants of maximal ideal space of a Banach algebra, Advances in Math. 19 (1976), 149-206.

[37] E.C. Titchmarsh, The Theory of Functions (Second Edition) (Oxford University Press, 1939).

[38] E.C. Titchmarsh, Introduction to the theory of Fourier integrals (third edition) (Oxford University Press, 1986).

[39] C.A. Tracy and H. Widom, Level spacing distributions and the Bessel kernel, Comm. Math. Phys. 161 (1994), 289-309.

[40] C.A. Tracy and H. Widom, Fredholm determinants, differential equations and matrix models, Comm. Math. Phys. 163 (1994), 33-72.

[41] K. Triméche, Transformation intégrale de Weyl et théorème de Paley-Wiener associés à un opérateur différentiel singulier sur $(0, \infty)$, J. Math. Pures Appl. 60 (1981), 51-98. 